\title{
Article
}

\section{Reparametrization Invariance and Some of the Key Properties of Physical Systems}

\author{
Vesselin G. Gueorguiev ${ }^{1,2, *(1)}$ and Andre Maeder $^{3}$ (i) \\ 1 Institute for Advanced Physical Studies, Sofia 1784, Bulgaria \\ 2 Ronin Institute for Independent Scholarship, 127 Haddon Pl., Montclair, NJ 07043, USA \\ 3 Geneva Observatory, University of Geneva, Chemin des Maillettes 51, CH-1290 Sauverny, Switzerland; \\ andre.maeder@unige.ch \\ * Correspondence: Vesselin@MailAPS.org
}

Citation: Gueorguiev, V.G.;

Maeder, A. Reparametrization Invariance and Some of the Key Properties of Physical Systems. Symmetry 2021, 13, 522. https:// doi.org/10.3390/sym13030522

Academic Editor: Rami Ahmad El-Nabulsi

Received: 28 February 2021

Accepted: 22 March 2021

Published: 23 March 2021

Publisher's Note: MDPI stays neutral with regard to jurisdictional claims in published maps and institutional affiliations.

Copyright: (C) 2021 by the authors. Licensee MDPI, Basel, Switzerland. This article is an open access article distributed under the terms and conditions of the Creative Commons Attribution (CC BY) license (https:// creativecommons.org/licenses/by/ $4.0 /)$.
Abstract: In this paper, we argue in favor of first-order homogeneous Lagrangians in the velocities. The relevant form of such Lagrangians is discussed and justified physically and geometrically. Such Lagrangian systems possess Reparametrization Invariance (RI) and explain the observed common Arrow of Time as related to the non-negative mass for physical particles. The extended Hamiltonian formulation, which is generally covariant and applicable to reparametrization-invariant systems, is emphasized. The connection between the explicit form of the extended Hamiltonian $\boldsymbol{H}$ and the meaning of the process parameter $\lambda$ is illustrated. The corresponding extended Hamiltonian $\boldsymbol{H}$ defines the classical phase space-time of the system via the Hamiltonian constraint $\boldsymbol{H}=0$ and guarantees that the Classical Hamiltonian $H$ corresponds to $p_{0}$-the energy of the particle when the coordinate time parametrization is chosen. The Schrödinger's equation and the principle of superposition of quantum states emerge naturally. A connection is demonstrated between the positivity of the energy $E=c p_{0}>0$ and the normalizability of the wave function by using the extended Hamiltonian that is relevant for the proper-time parametrization.

Keywords: diffeomorphism invariant systems; reparametrization-invariant systems; Hamiltonian constraint; homogeneous singular Lagrangians; generally covariant theory; equivalence of the Lagrangian and Hamiltonian framework; Canonical Quantization formalism; extended phasespace; extended Hamiltonian framework; proper time and proper length; relativistic Hamiltonian framework; relativistic particle; Minkowski space-time physical reality; common Arrow of Time; non-negativity of the mass of particles; positivity of the rest energy; Schrodinger's equation; wavefunction normalization; superposition principle in Quantum Mechanics

PACS: 03.50.-z; 04.90.+e; 11.10.Ef; 11.90.+t; 04.20.Gz; 04.20.Fy; 04.60.Ds; 04.60.Gw; 24.10.Jv

\section{Introduction}

It has taken thousands of years for natural philosophers and thinkers to arrive at the law of inertia, to accept it, and to turn it into a useful scientific paradigm by overthrowing the "obvious" Aristotelian physics (due to Aristotle 384-322 BCE). The process has been slow and painful with occasional advances in its formulation and better understanding of the law of inertia by various predecessors of Newton, such as Avicenna (Ibn Sīnā 980-1037), Galileo (1564-1642), who formulated the law of inertia for horizontal movement on the Earth, and later generalized by Descartes in his "Discourse of the Method" (1637). Until it finally finds its place as Newton's first principle: an object maintains its state, of rest or constant velocity propagation through space, unless a force acts on it, along with the fertile company of the other two laws whose mathematical formulation has been a breakthrough in mechanics.

At first sight, such principle seems to be untrue due to our everyday experience which shows that for an object to maintain its constant velocity an external influence is needed. 
The accumulation of knowledge and technological progress have made it possible for Newton to find the framework and formulate the three main principles that are now the cornerstone of Newtonian Mechanics.

In Newtonian Mechanics, time is a parameter that all observers that are connected via Galilean transformations will find to be the same-as long as they use the same identical clocks to keep a record of their time. The Galilean transformations are reflecting the symmetry under which the lows of the Newtonian Mechanics are form-invariant [1,2]. The spatial coordinates of the processes studied may have different values for different inertial observers, but these observers can compare their observations and would find an agreement upon utilization of the Galilean transformations. In this sense, the time coordinate is disconnected/disjoint from the configuration space $M$, which is used to label the states of the system/process, however, it is essential for the definition of the velocity vectors in the cotangent space TM.

In Special Relativity (SR), time becomes related to the observer and the Lorentz transformations intertwine space and time together in a Minkowski space-time [1,3,4]. This way the time duration of a process could be measured by different observers to be different even if they use identical laboratory clocks. However, all observers can identify a time duration related to an observer that is at rest with respect to the process's coordinate frame (co-moving frame). This is the proper-time duration of a process. Then all observers that are connected by Lorentz transformations will arrive at the same value for the propertime duration of a process. Special Relativity unifies the time coordinate with the spatial coordinates of an observer to a spacetime the configuration space of the coordinates of events. This way, from the point of view of an observer, the space-time is divided into three important subsets: the time-like paths, space-like curves, and light-like paths or equivalently into a past and future cones inside the light-cone defined by the light-like paths connected to the observer, and the space-like exterior of the rest of space-time. Lorentz transformations preserve the local light-cone at any point in the space-time and thus the causal structure of the time-like paths describing a physical process.

General Relativity (GR) goes even further by allowing comparison between observers related by any coordinate transformations, as long as there is an equivalent local observer who's space-time is of Minkowski type. This means that time records associated with identical clocks that undergo arbitrary physically acceptable motion/process can be compared successfully - that is, the observers will reach a mutually acceptable agreement on what is going on when studying a causal process. In this framework, a larger class of observers, beyond those in Newtonian and Special Relativity frameworks, can connect their laboratory time duration of a process to the proper-time duration measured by an observer in a co-moving frame along the time-like process. The essential ingredient of GR is the invariance of the proper-time interval $d \tau$ and the proper-length interval $d l$; this is achieved by the notion of parallel transport that preserves the magnitude of a vector upon its transport to nearby points in the configuration space-time. The symmetry transformations of the space-time associated with this larger class of observers are the largest possible set the diffeomorphisms of the space-time coordinates.A theory that has such symmetry is called covariant theory.All modern successful theories in physics are build to be explicitly covariant $[5,6]$.

Considering the above view of describing physical reality, and in particular, that any physically acceptable observer can use their own coordinate time as parametrization for a physical process then it seems reasonable to impose the principle of reparametrization invariance along with the principle of the covariant formulation when constructing models of the physical processes [6-8]. This means that along with the laboratory coordinates that label the events in the local space-time of an observer, who is an arbitrary and therefore can choose the coordinates in any way suitable, for the description of a natural phenomenon within the means of the laboratory apparatus. The observer should also be free to choose an arbitrary parametrization of the process as long as it is useful for the process considered. As long as the formulation of the model is covariant then there would be a suitable 
diffeomorphism transformation between any two physical observers that will allow them to reach agreement on the conclusions drawn from the data. Thus the process is independent of the observer's coordinate frame. The reparametrization invariance of the process then means that the process is also independent, not only on the coordinate frame of the observer, but it is also independent on the particular choice of process parametrization selected by the observer who is studying the process. Formulating a covariant theory is well known in various sub-fields of physics, but if one embraces the principle of reparametrization invariance then there are at least three important questions to be addressed:

1. How do we construct such models?

2. What is the mathematical framework and what are the implications of such models?

3. What is the meaning/role of an arbitrarily time-parameter $\lambda$ for a particular process?

The first question, "How do we construct reparametrization invariant models?" has been already discussed, in general terms, by the authors in a previous publications $[6,7,9]$ along with further relevant discussions of the possible relations to other modern theories and models $[6,8,10]$. The important line of reasoning is that fiber bundles provide the mathematical framework for classical mechanics, field theory, and even quantum mechanics when viewed as a classical field theory. Parallel transport, covariant differentiation, and gauge symmetry are very important structures associated with fiber bundles [4,7]. When asking: "What structures are important to physics?", one should also ask: "Why one fiber bundle should be more 'physical' than another?", "Why does the 'physical' base manifold seems to be a four-dimensional Minkowski manifold?" [6,7,11-13], and "How should one construct an action integral for a given fiber bundle?" [6,14-18]. Starting with the tangent or cotangent bundle seems natural because these bundles are related to the notion of classical point-like objects. Since we accumulate and test our knowledge via experiments that involve classical apparatus, the physically accessible fields should be generated by matter and should couple to matter as well. Therefore, the matter Lagrangian should contain the interaction fields, not their derivatives, with which classical matter interacts $[7,10,19]$. The important point here is that probing and understanding physical reality goes through a classical interface that shapes our thoughts as classical causality chains. Therefore, understanding the essential mathematical constructions in classical mechanics and classical field theory is important, even though quantum mechanics and quantum field theory are regarded as more fundamental than their classical counterparts.

In particular, the results relevant to Question 1 and 2 above seems to justify the existence only of electromagnetic and gravitational interactions, as we know them, at a classical level within the Lagrangian framework [9].

Two approaches, the Hamiltonian and the Lagrangian framework, are very useful in physics $[2,14,15,20-23]$. In general, there is a transformation that relates these two approaches. For a reparametrization-invariant theory $[9,21,22,24-26]$, however, there are problems in changing from Lagrangian to the Hamiltonian approach $[2,20-23,27,28]$.

Given the remarkable results in [9] due to the idea of reparametrization invariance, it is natural to push the paradigm further and to address point 2 above, and to seek a suitable Hamiltonian formulation along with a relevant quantum framework. Some of the problems faced by reparametrization invariant systems studied here are also relevant to string theory and general relativity. In this respect the lessons learned could be relevant to the understanding of space, time, and the quantum phenomenon [29].

In this paper, the problems related to changing from Lagrangian to the Hamiltonian approach are illustrated and their resolution for the simplest one-dimensional reparametrization-invariant systems relevant to the physical reality as well as in the case of the relativistic particle in any dimension are discussed.

The relativistic particle Lagrangian is used to justify the importance of reparametrizationinvariant systems and in particular the first-order homogeneous Lagrangians in the velocities. The usual gravitational interaction term along with the observational fact of finite propagational speed is used to justify the Minkowski space-time physical reality. The justification implies only one time-like coordinate in addition to the spatial coordinates 
along which particles propagate with a finite speed. By using the freedom of choosing time-like parametrization for a process, it is argued that the corresponding causal structure results in the observed common Arrow of Time and non-negative masses for the physical particles. The meaning of the time parameter $\lambda$ is further investigated within the framework of reparametrization-invariant systems. Such systems are studied from the point of view of the Lagrangian and extended Hamiltonian formalism. The extended Hamiltonian formulation is using an extended Poisson bracket $\llbracket, \rrbracket$ which is generally covariant and applicable to reparametrization-invariant systems. The extended Poisson bracket $\llbracket$, $\rrbracket$ is defined over the extended phase-space (phase-space-time) and includes the coordinate time $t$ and the energy $p_{0}$ in a way consistent with the Canonical Quantization formalism. The corresponding extended Hamiltonian $\boldsymbol{H}$ defines the classical phase space-time of the system via the Hamiltonian constraint $\boldsymbol{H}=0$ and guarantees that the Classical Hamiltonian $H$ corresponds to $c p_{0}$ the energy of the particle when the parametrization $\lambda=c t$ is chosen. Furthermore, if the extended Hamiltonian for a classical system is quantized $(\boldsymbol{H} \rightarrow \hat{\boldsymbol{H}})$ by following the Canonical Quantization formalism and the corresponding Hilbert space $\mathcal{H}$ is defined via the extended Hamiltonian $\hat{H} \Psi=0$ then the Schrödinger's equation emerges naturally and the principle of superposition of quantum states is justified. A connection is demonstrated between the positivity of the energy $E=c p_{0}>0$ and the normalizability of the wave function by using the extended Hamiltonian that is relevant for the proper-time parametrization. It is demonstrated that the choice of the extended Hamiltonian $\boldsymbol{H}$ is closely related to the meaning of the process parameter $\lambda$. The two familiar roles that $\lambda$ can take upon the coordinate time $t$ and the proper-time $\tau$ are illustrated using the simplest one-dimensional reparametrization invariant systems. In general, $\lambda$ can also be the proper length along the path of a particle for appropriately chosen transformation generator similar to $\boldsymbol{H}$.

The formalism is further illustrated in more details for the case of the relativistic particle in Appendix A section of the paper.

The discussion starts by first reviewing the main points from [6-9] as pertained to point particles: Section 2 has a Section 2.1 on the Lagrangian for a relativistic particle as an example of a reparametrization-invariant system, followed by a Section 2.3 where the general properties of homogeneous Lagrangians in the velocities are stated, the section concludes with a list of pros and cons of the first-order homogeneous Lagrangians. The next Section 3 is revisiting the argument why a space-time with a maximum speed of propagation through space, when modeled via first-order homogeneous Lagrangian based on a metric tensor, should be locally a Minkowski space-time with common Arrow of Time and non-negative mass for the particles. Section 4 is a brief review of Lagrangian and Hamiltonian Mechanics and the problem of the Hamiltonian constraint $H=0$ for systems based on first-order homogeneous Lagrangians. In Section 5, the Canonical Quantization is used as justification for the introduction of the extended covariant Hamiltonian framework within which the Hamiltonian constraint $\boldsymbol{H}=0$ can be used to define the phase space-time, as well as to justify the Schrödinger's equation as a consequence of applying Canonical Quantization to the extended Hamiltonian framework. The meaning of the process parameter $\lambda$ within the extended Hamiltonian framework is discussed in Section 6 using the simplest possible one-dimensional reparametrization invariant systems. The conclusions and discussions are given in Section 7. The formalism is illustrated in more details for the case of the relativistic particle in the Appendix A.

\section{Justifying the Reparametrization Invariance (RI)}

\subsection{Relativistic Particle Lagrangian}

From everyday experience, we know that localized particles move with a finite speed in a three-dimensional space. However, in an extended-configuration space (space-time), when the time is added as a coordinate $\left(x^{0}=c t\right)$, massive particles move along a spacetime trajectory such that $u \cdot u=1$. Here, $u^{\mu}$ are the coordinates of a general 4-velocity vector $v^{\mu}=d x^{\mu} / d \lambda$ but with a special choice of the parametrization parameter $\lambda$; that is, 
$u^{\mu}=d x^{\mu} / d \tau$. While $\lambda$ is an arbitrary parametrization, $\tau$ is a special choice of parametrization that is invariant with respect to any coordinate transformations between reasonable physical observers, it is the proper-time $(\tau)$ mathematically defined via a metric tensor $g_{\mu \nu}$ $\left(d \tau^{2}=g_{\mu \nu} d x^{\mu} d x^{v}\right)$. In particular when the metric tensor takes the form of the Minkowski metric $\left(g_{\mu \nu}=\eta_{\mu v}\right)$ then one can talk about local Lorentz equivalent observers. In this case, the action for a massive relativistic particle has a nice geometrical meaning: the "time distance" along the particle trajectory [4]:

$$
\begin{aligned}
& S_{1}=\int d \lambda L_{1}(x, v)=\int d \lambda \sqrt{g_{\mu v} v^{\mu} v^{v}}, \\
& \sqrt{g_{\mu \nu} v^{\mu} v^{v}} \rightarrow \sqrt{g_{\mu v} u^{\mu} u^{v}}=1 \Rightarrow S_{1} \rightarrow \int d \tau .
\end{aligned}
$$

However, for massless particles, such as photons, the 4-velocity is a null vector $\left(g_{\mu v} v^{\mu} v^{v}=0\right)$. Thus, proper time is not well defined and furthermore, one has to use a different Lagrangian to avoid problems due to division by zero when evaluating the final Euler-Lagrange equations. The appropriate "good" action is then [4]:

$$
S_{2}=\int L_{2}(x, v) d \lambda=\int g_{\mu v} v^{\mu} v^{v} d \lambda .
$$

For a massive particle, the Euler-Lagrange equations obtained from $S_{1}$ and $S_{2}$ are equivalent, this equivalence is discussed in more detail later. In the above discussion, it has been considered an arbitrary parametrization $\lambda$ and the proper-time parametrization $\tau$ for a massive particle. The physical meaning of the proper-time $\tau$ is usually considered to be the passing of clock time of a co-moving observer. Another important parametrization is the coordinate time $t$ corresponding to the clock time of an arbitrary physical observer that is studying the motion of the massive particle. Contemporary physics models are expected to be invariant with respect to coordinate transformations between physical observers. This is achieved by constructing Lagrangians as a scalar object from various vector and tensor quantities that correspond to the measurements of an arbitrary observer. In mathematical terms, this is a diffeomorphism invariance of the coordinate space. Thus, the physics content of a process under study is the same and therefore independent of the coordinate system of an observer. Clearly, diffeomorphism invariance is an important symmetry that reflects the expectation that observing a process should not affect the process itself. Thus, various observers should find a way to understand each-others measurements in a consistent way as long as they pertain to the same process under the study.

In the example above, the process under study is the motion of a massive particle. In this respect, the process corresponds to a one-dimensional manifold that is a curve in a higher dimensional space-time. It seems that the relationships of the points along the curve, in particular, the ordering of the points and their relative measures, should be something about the curve (the process under study). Thus, various observers should be able to find a consistent way to understand the curve and its properties. Therefore, the description of the curve should be independent of the choices an observer can make in order to describe the curve. In particular, the choice of parametrization of the curve should be irrelevant to the understanding of the corresponding process. Thus, a reparametrization invariant formulation would be the corresponding symmetry that the description should obey. While a model built on $L_{1}$ above does obey such symmetry, its quadratic version based on $L_{2}$ does not seem to obey it, even though the corresponding Euler-Lagrange equations are equivalent. Even more, the Euler-Lagrange equation does obey parametrization-rescaling symmetry that is easily seen when the Lagrangian is a homogeneous function of the velocity (see below). A way to resolve this puzzle is to recognize that $L_{2}$ can be viewed as a reparametrization invariant Lagrangian in a particular fixed gauge [30]:

$$
S_{2}^{\prime}=\int g_{\mu v} v^{\mu} v^{v} e^{-1} d \lambda .
$$


Here $e$ is an auxiliary field that makes the action $S_{2}^{\prime}$ reparametrization invariant by choosing $e \rightarrow \tilde{e}=e d \lambda / d \tilde{\lambda}$ when $\lambda \rightarrow \tilde{\lambda}$. Since now $S_{2}^{\prime}$ is reparametrization invariant then one can choose a gauge parametrization such that $e=1$ and thus arriving at $S_{2}$ but under propertime parametrization $\lambda=\tau$ where $g_{\alpha \beta} u^{\alpha} u^{\beta}=1$. Having to choose the gauge such that $g_{\alpha \beta} v^{\alpha} v^{\beta}$ is constant guarantees the equivalence of $L_{1}$ and $L_{2}[9,31]$.

\subsection{Equivalence of Homogeneous Lagrangians}

The above equivalence of $S_{1}$ and $S_{2}$ could be demonstrated on a more complicated Lagrangian as a specific choice of parametrization such that $g_{\alpha \beta}(x) v^{\alpha} v^{\beta}$ is constant $[9,31]$. Indeed, if one starts with the reparametrization invariant Lagrangian:

$$
L=q A_{\alpha} v^{\alpha}-m \sqrt{g_{\alpha \beta}(x) v^{\alpha} v^{\beta}}
$$

and defines proper-time gauge $\tau$ such that:

$$
d \tau=\sqrt{g_{\alpha \beta} d x^{\alpha} d x^{\beta}} \Rightarrow \sqrt{g_{\alpha \beta} u^{\alpha} u^{\beta}}=u \cdot u=1,
$$

then one can effectively consider

$$
L=q A_{\alpha} u^{\alpha}-(m-\chi) \sqrt{g_{\alpha \beta} u^{\alpha} u^{\beta}}-\chi
$$

as the model Lagrangian. Here $\chi$ is a Lagrange multiplier to enforce $u \cdot u=1$ in (5) that breaks the reparametrization invariance explicitly. Then one can write it as:

$$
L=q A_{\alpha} u^{\alpha}-(m-\chi) \frac{g_{\alpha \beta} u^{\alpha} u^{\beta}}{\sqrt{g_{\alpha \beta} u^{\alpha} u^{\beta}}}-\chi
$$

and using $u \cdot u=1$ in (5) one arrives at:

$$
L=q A_{\alpha} u^{\alpha}-(m-\chi) g_{\alpha \beta} u^{\alpha} u^{\beta}-\chi .
$$

One can deduce a specific value for $\chi(\chi=m / 2)$ by requiring that (4) and (6) produce the same Euler-Lagrange equations under the constraint $u \cdot u=1$ in (5). Then, by dropping the overall constant term, this finally results in the familiar equivalent Lagrangian:

$$
L=q A_{\alpha} u^{\alpha}-\frac{m}{2} g_{\alpha \beta} u^{\alpha} u^{\beta} .
$$

where $\tau$ has the usual meaning of proper-time parametrization such that $u \cdot u=1$ in (5), but it is imposed after deriving all the equations from the Lagrangian under consideration.

The equivalence between $S_{1}$ and $S_{2}$ is very robust. Since $L_{2}$ is a homogeneous function of order 2 with respect to the four-velocity $\vec{v}$, the corresponding Hamiltonian function $(H=v \partial L / \partial v-L)$ is exactly equal to $L(H(x, v)=L(x, v))$. Thus, $L_{2}$ is conserved, and so is the length of $\vec{v}$ and therefore $L_{1}$ is conserved as well. Any homogeneous Lagrangian in $\vec{v}$ of order $n \neq 1$ is conserved because $H=(n-1) L$. When $d L / d \lambda=0$, then one can show that the Euler-Lagrange equations for $L$ and $\tilde{L}=f(L)$ are equivalent under certain minor restrictions on $f$ [9].

To see this, consider the Euler-Lagrange equation for $L$ :

$$
\frac{d}{d \lambda}\left(\frac{\partial L}{\partial v^{i}}\right)-\frac{\partial L}{\partial x^{i}}=0
$$

and compare it with the Euler-Lagrange equation for $\tilde{L}=f(L)$ that can be written as:

$$
\left(\frac{f^{\prime \prime}}{f^{\prime}} \frac{d L}{d \lambda}\right)\left(\frac{\partial L}{\partial v^{i}}\right)+\frac{d}{d \lambda}\left(\frac{\partial L}{\partial v^{i}}\right)-\left(\frac{\partial L}{\partial x^{i}}\right)=0
$$


Clearly, these equations will be equivalent if the Lagrangians $L$ and $\tilde{L}=f(L)$ are constants of the motion; that is, $d L / d \lambda=0$, and $f^{\prime}$ and $f^{\prime \prime}$ are well behaved.

This is an interesting type of equivalence that applies to homogeneous Lagrangians $\left(L(\beta v)=\beta^{n} L(v)\right)$. It is different from the usual equivalence $L \rightarrow \widetilde{L}=L+d \Lambda / d \lambda$ or the more general equivalence discussed in Reference $[18,20,32,33]$. Any solution of the EulerLagrange equation for $\tilde{L}=L^{\alpha}$ would conserve $L=L_{1}$ since $\tilde{H}=(\alpha-1) L^{\alpha}$ when $\alpha \neq 1$, while for $\alpha=1$ it can be enforced as a choice of parametrization. For example, as demonstrated above, one can always make the choice of proper-time parametrization $\lambda=\tau$ for a massive particle. All these solutions are solutions of the Euler-Lagrange equation for $L$ as well; thus, $L^{\alpha} \subset L$. The fact that the models based on $L_{2}$ are only a subset of $L_{1}$ implies that $L_{1}$ has a special role due to its richer applicability to physical systems. In particular, such Lagrangians are of unique type relevant to the Weyl Integrable geometry [34]. Weyl's Integrable geometry provides a framework that is likely to be relevant to physics [35] and may have a far reaching consequences for cosmology [36]. In general, conservation of $L_{1}$ is not guaranteed since $L_{1} \rightarrow L_{1}+d \Lambda / d \lambda$ is also a homogeneous Lagrangian of order one equivalent to $L_{1}$. This suggests that there may be a choice of $\lambda$, a "gauge fixing", such that $L_{1}+d \Lambda / d \lambda$ is conserved even if $L_{1}$ is not. The above discussion applies to any homogeneous Lagrangian that has no explicit time dependence.

\subsection{Homogeneous Lagrangians of First Order}

Suppose we know nothing about classical physics, which is mainly concerned with trajectories of point particles in some space $M$, but we are told we can derive it from a variational principle if we use the right action integral $S=\int L d \lambda$. By following the above example we wonder: "should the smallest 'distance' be the guiding principle?" when constructing $L$. If yes, "How should it be defined for other field theories?" It seems that a reparametrization-invariant theory can provide us with a metric-like structure $[17,27,28,37]$, and thus a possible link between field models and geometric models [38].

In the example of the relativistic particle, the Lagrangian and the trajectory parameterization have a geometrical meaning. In general, however, parameterization of a trajectory is quite arbitrary for any observer. If there is such thing as the smallest time interval that sets a space-time scale, then this would imply a discrete space-time structure since there may not be any events in the smallest time interval. The Planck scale is often considered to be such a special scale [39,40]. Leaving aside hints for quantum space-time from loop quantum gravity and other theories, we ask: "Should there be any preferred trajectory parameterization in a smooth 4-dimensional space-time?" and "Are we not free to choose the standard of distance (time, using natural units $c=1$ )?" If so, then one should have a smooth continuous manifold and the theory should not depend on the choice of parameterization.

If one examines the Euler-Lagrange equations carefully:

$$
\frac{d}{d \lambda}\left(\frac{\partial L}{\partial v^{\alpha}}\right)=\frac{\partial L}{\partial x^{\alpha}},
$$

one will notice that any homogeneous Lagrangian of order $n\left(L(x, \alpha \vec{v})=\alpha^{n} L(x, \vec{v})\right)$ provides a reparametrization invariance of the equations under the rescaling transformations of the parametrization $\lambda \rightarrow \lambda / \alpha, \vec{v} \rightarrow \alpha \vec{v}$. Next, note that the action $S$ involves an integration that is a natural structure for orientable manifolds $(M)$ with an $n$-form of the volume. Since a trajectory is a one-dimensional object, then what is one looking at is an embedding $\phi: \mathbb{R}^{1} \rightarrow M$. This means that one is pushing forward the tangential space $\phi_{*}: T\left(\mathbb{R}^{1}\right)=$ $\mathbb{R}^{1} \rightarrow T(M)$, and is pulling back the cotangent space $\phi^{*}: T\left(\mathbb{R}^{1}\right)=\mathbb{R}^{1} \leftarrow T^{*}(M)$. Thus, a 1-form $\omega$ on $M$ that is in $T^{*}(M)\left(\omega=A_{\mu}(x) d x^{\mu}\right)$ will be pulled back on $\mathbb{R}^{1}\left(\phi^{*}(\omega)\right)$ and there it should be proportional to the volume form on $\mathbb{R}^{1}\left(\phi^{*}(\omega)=A_{\mu}(x)\left(d x^{\mu} / d \lambda\right) d \lambda \sim d \lambda\right)$, allowing it to be integrated $\int \phi^{*}(\omega)$ :

$$
\int \phi^{*}(\omega)=\int L d \lambda=\int A_{\mu}(x) v^{\mu} d \lambda .
$$


Therefore, by selecting a 1-form $\omega=A_{\mu}(x) d x^{\mu}$ on $M$ and using $L=A_{\mu}(x) v^{\mu}$ one is actually solving for the embedding $\phi: \mathbb{R}^{1} \rightarrow M$ using a chart on $M$ with coordinates $x: M \rightarrow \mathbb{R}^{n}$. The Lagrangian obtained this way is homogeneous of first-order in $v$ with a very simple dynamics. The corresponding Euler-Lagrange equation is $F_{v \mu} v^{\mu}=0$ where $F$ is a 2-form $(F=d A)$; in electrodynamics, this is the Faraday's tensor. If one relaxes the assumption that $L$ is a pulled back 1-form and assume that it is just a homogeneous Lagrangian of order one, then one may find a reparametrization-invariant theory that could have an interesting dynamics. The above mathematical reasoning can be viewed as justification for the known classical forces of electromagnetism and gravitation and perhaps even of new classical fields beyond electromagnetism and gravitation $[6,7,9,10]$.

\subsection{Pros and Cons of Homogeneous Lagrangians of the First Order}

Although most of the features listed below are more or less self-evident, it is important to compile a list of properties of the first-order homogeneous Lagrangians in the velocity $\vec{v}$. Some of the good properties of a theory with a first-order homogeneous Lagrangian are [9]:

(1) First of all, the action $S=\int L\left(x, \frac{d x}{d \lambda}\right) d \lambda$ is a reparametrization invariant.

(2) For any Lagrangian $L\left(x, v=\frac{d x}{d t}\right)$ one can construct a reparametrization-invariant Lagrangian by enlarging the configuration space $\{x\}$ to an extended configuration space-the space-time $\{c t, x\}[2,21,22]$. However, it is an open question whether there is a full equivalence of the corresponding Euler-Lagrange equations.

(3) Parameterization-independent path-integral quantization could be possible since the action $S$ is reparametrization invariant [41].

(4) The reparametrization invariance may help in dealing with singularities [26].

(5) It is easily generalized to $D$-dimensional extended objects ( $p$-branes / $d$-branes) [6,7].

The list of trouble-making properties in a theory with a first-order homogeneous Lagrangian includes:

(1) There are constraints among the Euler-Lagrange equations $[2,22]$, since $\operatorname{det}\left(\frac{\partial^{2} L}{\partial v^{\alpha} \partial v^{\beta}}\right)=0$.

(2) It follows that the Legendre transformation $\left(T(M) \leftrightarrow T^{*}(M)\right)$, which exchanges velocity and momentum coordinates $(x, v) \leftrightarrow(x, p)$, is problematic [20].

(3) There is a problem with the canonical quantization approach since the Hamiltonian function is identically ZERO $(H \equiv 0)[23,28]$,

The pro (2) and the con (3) above are of key importance. The procedure that can be utilized as mentioned in pro (2) above is very simple: $L\left(x, \frac{d x}{d t}\right) \rightarrow \dot{t} L\left(x, \frac{\dot{x}}{\dot{t}}\right)$ where the dotted notation is a derivative with respect to the parametrization $\lambda$, that is, $\dot{t}=\frac{d t}{d \lambda}$ and $\dot{x}=\frac{d x}{d \lambda}[21,22]$. This means that every Lagrangian based theory can be reformulated in a reparametrization invariant form. This is a different symmetry from the diffeomorphism invariance of the theory, which is still satisfied by construction. However, the parameter $\lambda$ does not have to be the typical physical time parameterization of a process like its own process time-the proper time $\tau$, nor the coordinate time $t$ for the observer that is studying the process. In this sense, $\lambda$ could be truly arbitrary and thus demonstrating the existence of a larger class of theories that do satisfy the principle of reparametrization invariance as discussed in the introduction. The problem with these larger class of theories is in the con (3). Which makes the standard quantization treatment quite difficult and unusual due to the presence of constraints among the equations of motion con (1) above [21]. In this paper, we are mostly concerned with physical processes that are associated with one-dimensional manifolds and their reparametrization. However, as the pro (5) suggests, the formulation is relevant to two-dimensional sub-manifolds, which is the domain of string theory, and extends to high-dimensional sub-manifolds with reparametrization invariant Lagrangians such as the Nambu-Gotto Lagrangians [10,23]. The reparametrization invariance, which is a diffeomorphism of the submanifold corresponding to a physical process, is a farreaching idea and it is different from the coordinate diffeomorphisms of the target space [9]. However, it is beyond the scope of this paper to go into string-theory, p-branes, and gravity that represent sub-manifolds with dimension bigger than one. 
Constraints among the equations of motion are not an insurmountable problem since there are procedures for quantizing such theories [23,42-46]. For example, instead of using $H \equiv 0$ one can use some of the constraint equations available, or a conserved quantity, as Hamiltonian for the quantization procedure [23]. Changing coordinates $(x, v) \leftrightarrow(x, p)$ seems to be difficult, but it may be resolved in some special cases by using the assumption that a gauge $\lambda$ has been chosen so that $L \rightarrow L+\frac{d \Lambda}{d \lambda}=\tilde{L}=$ const. The above-mentioned quantization troubles will not be discussed here since they are outside of the scope of this paper. A new approach that resolves $H \equiv 0$ and naturally leads to a Dirac-like equation is under investigation, for some preliminary details see Reference [6]. Here, the focus is on the understanding of the meaning and the role of the general parameter $\lambda$ by extending the Hamiltonian framework to an extended phase-space (phase-space-time) with a covariant

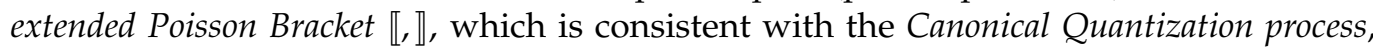
along with an extended Hamiltonian $\boldsymbol{H}$ that defines the extended phase-space-time via $\boldsymbol{H} \equiv 0$. By following the Canonical Quantization formalism $(\boldsymbol{H} \rightarrow \hat{\boldsymbol{H}})$ the Hilbert space of the quantum system can be defined via the corresponding extended Hamiltonian $\hat{H}$ as the linear space of states $\Psi$ that satisfy $\hat{H} \Psi=0$. As a byproduct, one can use this formulation to justify the Schrödinger's equation. A similar approach to the Schrödinger's equation has been discussed in Reference [21,22,47]. Furthermore, we do not concern ourselves with the questions about the algebra of observables nor with issues of unboundedness of important physical operators or alternative approaches to the quantization framework. Such issues are outside of the scope of the current paper. Finally, before we discuss the extended Hamiltonian framework, we would like to use the structure of the first-order homogeneous Lagrangians to mathematically justify few other important features of the physical reality that we often take for granted.

\section{One-Time Physics, Causality, Arrow of Time, and the Maximum Speed of Propagation}

In our everyday life, most of us take time for granted, but there are people who are questioning its actual existence or consider models with more than one time-like coordinate $[1,11,12,48-65]$. Since we are trying to understand the meaning of an arbitrary time-like parameter $\lambda$ within the framework of reparametrization invariant systems, it seems important to think about the possible number of time-parameters. Here, we briefly argue that a one-time-physics, in case of massive point particles, is essential to assure causality via finite propagational speed through space for such massive point particles [6]. Then the common arrow of time, which is often viewed as related to the increasing entropy as commanded by second law of thermodynamics $[55,66-71]$, becomes instead a consequence of the positivity of the rest mass.

\subsection{One-Time Physics, Maximum Speed of Propagation, and the Space-Time Metric Signature}

Why the space-time seems to be one time plus three spatial dimensions have been discussed by using arguments a la Wigner [11,12]. However, these arguments are deducing that the space-time is $1+3$ because only this signature is consistent with particles with finite spin. However, one should turn this argument backwards claiming that one should observe only particles with finite spin because the signature is $1+3$. A thermodynamic selection principle of the $(1+3)$ nature of the universe has been recently discussed [72]. And yet, there is an alternative argument for the emergence of the apparent Lorentzian dynamics of the usual field theories due to a scalar clock field that is playing the role of the physical time [73]. Furthermore, there is an argument for $(1+3)$ nature of the universe based on four fundamental principles of physics namely: Causality, General Covariance, Gauge Invariance, and Renormalizability [74]. This can be taken even further to dynamically generate a fifth dimension [75], which contains an extra special dimension in contrast to the traditional arguments for three special dimensions [76,77]. 
Here we revisit the argument that only one-time physics is consistent with a finite spatial propagational speed [6]. The local Lorentz symmetry implies the existence of a local observer with Minkowski like coordinate frame.

The main assumptions are:

I. Gravity-like term $\sqrt{g(\vec{v}, \vec{v})}$ is always present in the matter Lagrangian.

II. The corresponding matter Lagrangian is a real-valued function.

This way, physical processes, like propagation of a particle, must be related to positivevalued term $g(\vec{v}, \vec{v}) \geq 0$. Here $\vec{v}$ is the rate of change of the space-time coordinates with respect to some arbitrarily chosen parameter $\lambda$ that describes the evolution of the process (propagation of a particle). That is, $v^{\alpha}=d x^{\alpha} / d \lambda$. By speed one should mean the magnitude of the spatial velocity with respect to a laboratory time coordinate $x^{0}$ where $v_{\text {space }}^{i}=d x^{i} / d x^{0}$.

The use of a covariant formulation allows one to select a local coordinate system so that the metric is diagonal $(+,+, . .,+,-, \ldots-)$. If one denotes the $(+)$ coordinates as time coordinates and the $(-)$ as spatial coordinates, then there are three essential cases:

(1) No time coordinates will contradict $g(\vec{v}, \vec{v}) \geq 0$ :

$$
g(\vec{v}, \vec{v})=-\sum_{\alpha}\left(v^{\alpha}\right)^{2}<0
$$

(2) Two or more time coordinates-unconstrained spacial velocity $\vec{v}_{\text {space }}$ :

$$
g(\vec{v}, \vec{v})=\left(v^{0}\right)^{2}+\left(v^{1}\right)^{2}-\sum_{\alpha=2}^{n}\left(v^{\alpha}\right)^{2} \Rightarrow 1+w^{2} \geq \vec{v}_{\text {space }}^{2} .
$$

(3) Only one time coordinate enforces finite spacial velocity $\vec{v}_{\text {space }}$ :

$$
g(\vec{v}, \vec{v})=\left(v^{0}\right)^{2}-\sum_{\alpha=1}^{n}\left(v^{\alpha}\right)^{2} \Rightarrow 1 \geq \vec{v}_{\text {space }}^{2}
$$

Clearly, for two or more time coordinates, one does not have finite coordinate velocity $\left(\vec{v}_{\text {space }}^{2}=\left(d l / d x^{0}\right)^{2} \rightarrow \vec{v}^{2} / c^{2}\right)$ bound from above by the speed of light $c$. For example, when the coordinate time $\left(x^{0}\right)$ is chosen so that $x^{0}=c t \Rightarrow v^{0}=1$ then along another time-like coordinate $x^{1}$ the speed will be $w^{2}=\left(d x^{1} /(c d t)\right)^{2}$, which could be anything in magnitude unless $d x^{1} /(c d t)$ has an upper bound. Therefore, if $w$ is not zero then there would be processes that will exhibit deviations from the observed maximal speed $c$ of propagation. Thus, only the space-time with only one time accounts for a strict finite spatial speed of propagation, as observed, where the finite spatial velocity is bounded from above by the speed of light $v<c$. Therefore, a causal structure! When going from one point of space to another, it takes time and thus there is a natural causal structure $[63,78]$. The details of the causal structure will depend on the interactions that can take place when two objects are at the same point in the space-time since there is a natural future-and-past cone in such space-time.

\subsection{Causality, the Common Arrow of Time, and the Non-Negativity of the Mass}

In the previous section, we deduced that the space-time metric has to reflect that there is only one coordinate time and the rest of the coordinates should be spatial which is a requirement for finite spatial speed of propagation that induces causality. If nature is really reparametrization-invariant, then any observer studying a process can use its own time coordinate $t$ or any other suitable time-parameter $\lambda$, to label an unfolding process. However, when comparing to other observers who study the same process, it will be more advantageous to use a proper-time parametrization $\tau$ which is usually related to an observer who is following/moving along with the process (particle propagation in its 
co-moving frame). To be able to study a process using any laboratory time-coordinate $t$ and to deduce the process proper-time parametrization for the purpose of comparing to other arbitrary observers would then imply a reparametrization-invariant symmetry.

The process has to be related to a massive system because actual observers are also massive and cannot move as fast as light or other massless particles due to the previously deduced $1+n$ signature of the metric and non-negativity of $g(\vec{v}, \vec{v}) \geq 0$. As long as there is a term $m(v) \sqrt{g(\vec{v}, \vec{v})}$ in the Lagrangian $L$ and $m(0) \neq 0$ then the relationship $m(0) d \tau=m\left(v_{\text {space }}\right) \sqrt{g(\vec{v}, \vec{v})} d \lambda$ could be used to define proper-time $\tau$. Since $g(\vec{v}, \vec{v})>0$ then one can consider the positive branch of the square root function, otherwise upon utilizing the reparametrization symmetry one can consider $\lambda \rightarrow-\lambda$ that will correspond to the positive branch of the square root function when combined into $\sqrt{g(\vec{v}, \vec{v})} d \lambda$. Note that $m\left(v_{\text {space }}\right)$ is a homogeneous function of zero order since it depends only on the dimensionless special velocity $v^{i}=d x^{i} / d x^{0}=\left(d x^{i} / d \lambda\right) /\left(d x^{0} / d \lambda\right)$ that is invariant under reparametrization. If one considers the obvious choice $\lambda=t$ for any particular laboratory observer then this would imply that time is going forward for the observer as well as for the process-as long as $m(v) \neq 0$ for any physical value of $v$; thus, $m(v)$ and $m(0)$ have the same sign and therefore $m(v) / m(0)>0$. This would be valid for any two observers that can study each other's motion as well. Therefore, all observers that can study at least one common process in nature will find a common arrow of time $d t / d \tau=m(0) /(m(v) \sqrt{g(\vec{v}, \vec{v})})>0$. Such "positive flow" of time is important when considering the standard Lagrangian formulation for a relativistic particle and its reformulation as a reparametrization invariant system [21]. The "positive flow" of time assures the preservation of the sign of the mass term. Furthermore, by using the freedom of parametrization an observer may decide to use same process to define the scale of the time interval for the clocks. By choosing the parametrization to be the coordinate time with time intervals as measured by proper time intervals for a process, $d \lambda=d t=d \tau$, then this gives us the relation of the rest mass to moving mass $m(0)=m(v) \sqrt{g(\vec{v}, \vec{v})}$ as deduced in the theory of Special Relativity [4].

All processes and observers should have the same sign of their mass, if not then one can envision a non-interacting pair that has opposite sign of their rest masses $\left(m^{\prime}(0)=-m^{\prime \prime}(0)\right)$ moving in the same way (with $\vec{v}$ the same and thus $g(\vec{v}, \vec{v})$ the same as well) with respect to us; one expects that they will have the same proper-time; however, as a pair their propertime would not be accessible to us since their combined rest mass is zero $\left(m^{\prime}(0)+m^{\prime \prime}(0)=0\right)$. This situation creates a proper-time paradox if the two particles can be observed separately during the motion of the pair. This could well be the case of annihilating particle and antiparticle pair since then the notion of a proper-time of a photon is not well defined, however, a sub-system pair has never been observed as photon sub-structure. Given that all non-zero rest masses have to be of the same sign and the usual relationship between rest mass and energy $\left(E=m c^{2}\right)$, one can conclude that $m(v)>0$. Thus, the non-negativity of the masses of particles and the positivity of the mass of physical observers. One will see later that the positivity of the energy is related to the positivity of the norm of the corresponding quantum system in its proper-time quantization within the extended Hamiltonian formalism-see Section 6.2.3.

This is illustrated in more details in Appendix A for the case of the relativistic particle where the relativistic mass of a moving particle is related to the relativistic factor $\gamma$ and the rest mass of the particle $m(v)=\gamma(v) m(0)$.

In the light of the above discussion, the Common Arrow of Time is a result of the positivity of the mass and has nothing to do with the entropy of a closed system and the second law of thermodynamics [55,66-71].

To be more accurate one should point out that the positive correlation between forward-increasing time and the increasing entropy due to the second law of thermodynamics is not a cause-effect relation. Increasing entropy in general is just the general manifestation of forward-time flow. It is known that, locally, a system's entropy can be reduced but this does not change the flow of time. In our discussion above, the Common Arrow of Time is due to common processes between observers, "entanglement with the 
environment," which acts as a clock ([29], Chapter 17), which is forced to be synchronized due to the positivity of the mass. Thus, stopping and reversing the time flow for a macroscopic system is practically impossible, as it would have to overcome the second law of thermodynamics. However, for microscopic systems, that could be decoupled from the environment and the observer, the time-flow could be inverted, and therefore time-symmetric laws would be appropriate. Furthermore, there is a connection between the positivity of the mass and the positivity of the temperature in thermodynamics ([79], Chapter 1). This of course results, in general, in a decreasing of the temperature as entropy increases for a closed system, but this is the manifestation of the general tendency of decay towards the ground state of a system, which is not considered to be the cause of the Common Arrow of Time.

\section{From Lagrangian to Hamiltonian Mechanics}

In this section, we review the main relevant equations and concepts that we need to better understand the meaning of the parameter $\lambda$ in reparametrization-invariant systems and the particular roles it can take. To keep the notation simple we write the equations as for a one-dimensional system but the equations can easily be re-expressed for $n$-dimensional systems as well.

\subsection{Lagrangian Mechanics}

Given a Lagrangian $L(t, q, v)$, with velocity $v=\dot{q}=\frac{d q}{d t}$, one can derive the EulerLagrange equations of motion by minimizing the corresponding action $\mathcal{A}=\int L(t, q, \dot{q}) d t$ along trajectories $\{q(t): \delta A=0\}[4,17,27,28,37]$ :

$$
\frac{d p}{d t}=\frac{\partial L}{\partial q}
$$

where the generalized momentum $p$ is given as:

$$
p:=\frac{\partial L}{\partial v}
$$

If one considers the Hamiltonian function $H(t, q, v)$ :

$$
H=p v-L(q, v)
$$

then the Euler-Lagrange equations can be written as Hamilton's equations [15,27,46]:

$$
\begin{gathered}
v:=\frac{d q}{d t}=\frac{\partial H}{\partial p} \\
\frac{d p}{d t}=-\frac{\partial H}{\partial q}
\end{gathered}
$$

The full power of the Hamiltonian framework is realized if one can solve Equation (10) for all the velocities $v=d q / d t$ as functions of $(t, q, p)$; then one can study the system using $H(t, q, p)$ over the phase-space coordinates $(q, p)$. The dynamical equations of the Lagrangian framework (9) are then replaced by the dynamical equations of the Hamiltonian framework (13).

The rate of change of an observable $f(t, q, v)$ that is a function of time, position, and velocity is then given by:

$$
\frac{d f}{d t}=\frac{\partial f}{\partial q}\left(\frac{d q}{d t}\right)+\frac{\partial f}{\partial v}\left(\frac{d v}{d t}\right)+\frac{\partial f}{\partial t}
$$


In a similar way the rate of change of an observable over the phase-space $f(t, q, p)$ that is a function of time, position, and momentum is then given by:

$$
\frac{d f}{d t}=\frac{\partial f}{\partial q}\left(\frac{d q}{d t}\right)+\frac{\partial f}{\partial p}\left(\frac{d p}{d t}\right)+\frac{\partial f}{\partial t}
$$

When applied to the Hamiltonian function $H$ for solutions that satisfy the equations above, one obtains:

$$
\begin{aligned}
\frac{d H}{d t} & =\frac{\partial H}{\partial q}\left(\frac{d q}{d t}\right)+\frac{\partial H}{\partial p}\left(\frac{d p}{d t}\right)+\frac{\partial H}{\partial t}= \\
& =-\frac{\partial L}{\partial q} v+v \frac{d p}{d t}-\frac{\partial L}{\partial t}= \\
& =-\frac{d p}{d t} v+v \frac{d p}{d t}-\frac{\partial L}{\partial t}=-\frac{\partial L}{\partial t}
\end{aligned}
$$

Thus, if the Lagrangian does not depend explicitly on the time variable $t$ than the Hamiltonian function $H$ is conserved along any solution of the Euler-Lagrange equations.

\subsection{Hamiltonian Formalism}

The Hamiltonian Mechanics is based on a Hamiltonian function $H(t, q, p)$ over the phase-space coordinates $(q, p)$ along with a Poisson bracket $\{$,$\} which in its canonical$ form is:

$$
\{f, g\}=\frac{\partial f}{\partial q} \frac{\partial g}{\partial p}-\frac{\partial f}{\partial p} \frac{\partial g}{\partial q}=-\{g, f\}
$$

There are two sets of Hamilton equations of motion. The first set defines the rate of change (generalized velocity) of the coordinate function(s) $q$ :

$$
v:=\frac{d q}{d t}=\frac{\partial H}{\partial p}=\{q, H\},
$$

the second set is equivalent to the Euler-Lagrange equations:

$$
\frac{d p}{d t}=-\frac{\partial H}{\partial q}=\{p, H\}
$$

The rate of change of any observable $f(t, q, p)$ represented as a function over the phase space is then given by:

$$
\frac{d f}{d t}=\frac{\partial f}{\partial q}\left(\frac{d q}{d t}\right)+\frac{\partial f}{\partial p}\left(\frac{d p}{d t}\right)+\frac{\partial f}{\partial t}=\{f, H\}+\frac{\partial f}{\partial t}
$$

This expression, along with the antisymmetric property of the Poisson bracket (16), makes it easy to recognize that the Hamiltonian is conserved if $H$ does not depend explicitly on $t$.

\subsection{Problems with the Hamiltonian Function and the Legendre Transform for RI Systems}

The first problem that becomes clear when studying reparametrization-invariant systems based on first-order homogeneous Lagrangians is that the Hamiltonian function is identically zero [21,22]. The definition of a homogeneous function of order $n$ is $[1,10,18,32]$ :

$$
v \frac{\partial f(v)}{\partial v}=n f(v)
$$

Applying this to the Hamiltonian for a homogeneous Lagrangian of order $n$ in the velocities, one has: 


$$
H=v p-L=v \frac{\partial L}{\partial v}-L=(n-1) L
$$

Thus, the Hamiltonian will be identically zero for first-order homogeneous Lagrangians $(n=1)$ in the velocities:

$$
H=v p-L=v \frac{\partial L}{\partial v}-L \Rightarrow H \equiv 0 .
$$

Notice that this is independent of whether one is off-shell or on-shell (looking at solutions of the Euler-Lagrange equations of motion). It is just saying that $L_{1}=v p$. This problem can be mitigated if one considers equivalent Lagrangians. For example, $L_{(n)}=\left(L_{1}\right)^{n}$ will be a homogeneous function of order $n$. Thus, the issue $(H \equiv 0)$ will not occur since one has $H_{(n)}=(n-1) L_{(n)}$, which will be conserved if $L$ does not depend explicitly on the time coordinate $t$, implying conserved $L$ as well, then the Euler-Lagrange equations of motion related to various $L_{(n)}$ are equivalent to each other and correspond to the Euler-Lagrange equations of motion for $L_{1}$ :

$$
\begin{gathered}
\frac{d}{d t}\left(\frac{\partial L^{n}}{\partial v}\right)=\frac{\partial L^{n}}{\partial q} \Rightarrow \frac{d}{d t}\left(L^{(n-1)} \frac{\partial L}{\partial v}\right)=L^{(n-1)} \frac{\partial L}{\partial q^{\prime}} \\
\Rightarrow \frac{d}{d t}\left(\frac{\partial L}{\partial v}\right)+(n-1)\left(\frac{d}{d t} \ln (L)\right)\left(\frac{\partial L}{\partial v}\right)=\frac{\partial L}{\partial q}
\end{gathered}
$$

Therefore, solutions for $L_{(n)}=\left(L_{1}\right)^{n}$ are also solutions for $L_{1}$ and $L_{1}=v p$ will be conserved since $L_{(n)}$ is conserved. However, it is not guaranteed that all solutions for $L=L_{1}$ would result in the conservation of $L_{1}$.

Furthermore, going between Lagrangian and Hamiltonian formulation requires solving for $v$ in the Equation (10) or solving for $p$ in the Equation (17). When one has first-order homogeneous Lagrangian $L_{1}$ this is not possible because there are constraints in the system of equations and the Hessian matrix is singular $[20,32,43,46]$. How to resolve the constraints have been a subject of vast research for the purpose of achieving meaningful quantization $[19,24,42,44,71,80-83]$. We will not follow this path here and will consider an alternative approach to move forward towards a meaningful quantization. For this purpose, we now review the Canonical Quantization formalism [27,37,84].

\section{From Classical to Quantum Mechanics}

In this section, in order to briefly set the notions employed we follow the usual physics textbooks expositions of the quantization framework. Here, we are not concerned with the questions of boundness of the operators or alternative approaches to the quantization framework; these are important technical details but are not part of the scope of the current paper.

\subsection{Canonical Quantization}

In the standard Canonical Quantization, observables are replaced by operators $A \rightarrow \hat{A}$ over a Hilbert space $\mathcal{H}$, while the Poisson bracket is replaced by a commutator. Of course, this correspondence is not a strict equality since this would imply equality of quantum mechanics to Poissonian mechanics. Therefore, it is a functorial correspondence from the category of Poissonian systems to quantum systems where details depend on the specific systems considered:

$$
\{A, B\} \rightarrow \frac{1}{i \hbar}[\hat{A}, \hat{B}]
$$

Thus, the phase-space coordinates $q$ and $p$ are viewed as operators $\hat{q}$ and $\hat{p}$ satisfying the relation:

$$
\{q, p\}=1 \Rightarrow[\hat{q}, \hat{p}]=i \hbar
$$


In particular, if the Hilbert space $\mathcal{H}$ is taken to be the space of square integrable functions over the configuration space

$$
\mathcal{H}=\mathcal{L}_{2}\left[\psi(q): \hat{q} \psi(q)=q \psi(q), \int\|\psi(q)\|^{2}=1\right]
$$

then the momentum operator $\hat{p}$ becomes:

$$
\hat{p}=-i \hbar \frac{\partial}{\partial q}
$$

consistent with the notion that momentum is a generator of translations along the coordinate $q$.

The evolution of operators is now given by the Heisenberg equation, which is very similar to what one had in the Hamiltonian formalism [62]:

$$
\frac{d f}{d t}=\{f, H\}+\frac{\partial f}{\partial t} \rightarrow \frac{d \hat{f}}{d t}=\frac{1}{i \hbar}[\hat{f}, \hat{H}]+\frac{\partial \hat{f}}{\partial t}
$$

One shall not invoke yet the Schrödinger Equation since it will appear naturally in the approach presented. This way, one does not have to specify what $\hat{H}$ is either.

\subsection{Extending the Poisson Bracket}

Finding a covariant formulation of the Hamiltonian Mechanics has been an important topic of research leading to various approaches and methods. Here the aim is the extension of the configuration space to include time as a coordinate that allows us to connect to Quantum Mechanics. Thus, one needs to define a Hamiltonian and a suitable Poisson Bracket, and since the Hamiltonian formalism requires pairs of phase space-coordinates $(q, p)$ then one needs to identify the corresponding partner to the time coordinate $t$. From the theory of Special Relativity (SR), one already knows that it should somehow be related to the energy $E$, which is related to the $p_{0}$ component of a four-vector, and from Relativistic Quantum Mechanics (QM) and Relativistic Quantum Field Theory (QFT) one also has:

$$
\begin{gathered}
p_{0} \rightarrow \hat{p}_{0}=i \hbar \frac{\partial}{\partial t} \\
c \hat{p}_{0} \psi_{E}(t, q)=E \psi_{E}(t, q)
\end{gathered}
$$

However, measuring energy is also related to the Hamiltonian in Classical Mechanics as well as in Quantum Mechanics.

$$
E=H(q, p) \rightarrow E_{\psi}=<\psi|\hat{H}| \psi>
$$

In Quantum Mechanics the Hamiltonian is often the starting point to define a basis in the Hilbert space for the quantum system to be studied.

$$
\mathcal{H}=\mathcal{L}_{2}\left\{\psi(q)=£ c(E) \phi_{E}(q) d \mu_{E}: H \phi_{E}(q)=E \phi_{E}(q), \int d \mu_{E}\|\psi(E)\|^{2}=1\right\}
$$

However, if one starts with the first-order homogeneous Lagrangian, one has the problem of $H \equiv 0$, which is a general problem faced in other important reparametrization-invariant systems, such as String Theory and Quantum Gravity. In successful modern Quantum Field Theory models, the energy $E$ is proportional to $p_{0}$ as the generator of translations along the laboratory time coordinate (29) while the Hilbert space is determined from the relevant Relativistic Euler-Lagrange equations, such as the Dirac equation or its equivalent in the relevant Yang-Mills theory. So, it seems reasonable to give up on the notion that energy is related to a Hamiltonian operator coming from a classical system or that the basis of the Hilbert space can be associated to a Hamiltonian operator. Instead, it may be better to 
consider that $H \equiv 0$ should define the Hilbert space in a way similar to how one can derive the topology of a space by looking at the algebra of functions over this space.

We try to turn the issue $H \equiv 0$ into a virtue for some suitable expression of $\boldsymbol{H}$ that will define the Hilbert space.

$$
\mathcal{H}=\{\hat{\boldsymbol{H}} \psi \equiv 0\}
$$

This definition of the Hilbert space of a system clearly guarantees the principle of superposition of quantum states. Then, the evolution of the observables for the system (process) under a general parametrization $\lambda$ of a process studied:

$$
\frac{d \hat{f}}{d \lambda}=\frac{1}{i \hbar}[\hat{f}, \hat{\boldsymbol{H}}]
$$

should emerge from the extension of the Hamiltonian formalism.

If one extends the configuration space of a classical system from $q_{1}, \ldots, q_{n}$, to include $t$ as $q_{0}$, to $q_{0}, q_{1}, \ldots, q_{n}$ and the phase space by adding $p_{0}$ to the list of $(q, p)$ pairs of conjugated variables, then one should probably extend the Hamiltonian, so that the extension of the evolution Equation (19) would allow some meaningful interpretation. Furthermore, one would like the extension to be useful for reparametrization-invariant systems-so a general parametrization $\lambda$ should be used to determine the rate of change of an observable related to a function $f\left(q_{0}, q_{1}, \ldots, q_{n}, p_{0}, p_{1}, \ldots, p_{n}\right)$ :

$$
\begin{aligned}
& \frac{d f}{d t}=\{f, H\}+\frac{\partial f}{\partial t} \rightarrow \frac{d f}{d \lambda}=\llbracket f, H \rrbracket \\
\{f, g\}= & \frac{\partial f}{\partial q} \frac{\partial g}{\partial p}-\frac{\partial f}{\partial p} \frac{\partial g}{\partial q} \rightarrow \\
\rightarrow & \llbracket f, g \rrbracket=\{f, g\}+\left(\frac{\partial}{\partial x^{0}} \overleftrightarrow{\otimes} \frac{\partial}{\partial p_{0}}\right) \triangleright(f \overleftrightarrow{\otimes} g)
\end{aligned}
$$

This brings the attention only on the expression of the Poisson Bracket on the $\left(x^{0}, p_{0}\right)$ sub-space of the phase space.

One approach is to set $x^{0}=c t$ on a similar footing as all the other coordinates $q_{i}$ for $i=1 \ldots n$ (see for example Refs. $[21,22,28,85]$ ):

$$
\llbracket x^{0}, p_{0} \rrbracket=\left(\frac{\partial}{\partial x^{0}} \frac{\partial}{\partial p_{0}}-\frac{\partial}{\partial p_{0}} \frac{\partial}{\partial x^{0}}\right) \triangleright\left(x^{0} \otimes p_{0}\right)=1
$$

The rational for such a choice is to consider $q^{i}$ and $p_{j}$ as vectors living in dual spaces and thus the natural choice $\left\{q^{i}, p_{j}\right\}=\delta_{j}^{i}$ to be extended to $\llbracket q^{\mu}, p_{v} \rrbracket=\delta_{v}^{\mu}$ which leads to $\llbracket x^{0}, p_{0} \rrbracket=1$. The problem with this choice for the particular metric signature $\{+,-,-,-\}$, as discussed in Section 3.1, is that there is no difference between upper and lower 0 indexes. Thus, the coordinate time $t$ is too much like all the other configuration coordinates. As if done in treatments that are using the signature $\{-,+,+,+\}$, which is easy on the index manipulations for spacial vectors. Furthermore, there is no explicit local Lorentz invariance, and upon canonical quantization it does not give the usual expression for $\hat{p}_{0}$ that is more like the expression for the usual space-related momenta $\hat{p}_{i}$ (27) rather than (29). To resolve this issues, it seems better to consider:

$$
\{f, g\} \rightarrow \llbracket f, g \rrbracket=-\eta_{\mu \nu}\left(\frac{\partial f}{\partial q_{\mu}} \frac{\partial g}{\partial p_{\nu}}-\frac{\partial f}{\partial p_{\mu}} \frac{\partial g}{\partial q_{v}}\right)
$$

where $\mu=0,1, \ldots, n$ and $\eta_{\mu v}$ is the Lorentz-invariant tensor with signature $1,-1, \ldots,-1$. The minus sign in front of $\eta_{\mu v}$ is to recover the usual Poisson bracket between $q_{i}$ and $p_{i}$ for $i=1 \ldots n$ as expected in the signature $\{-,+,+,+\}$ treatment. This expression is explicitly 
Lorentz invariant, which singles out $q_{0}=c t$ as different from the other special coordinates $q_{i}$ and it results in the correct expression for $\hat{p}_{0}$ upon canonical quantization since now:

$$
\llbracket x^{0}, p_{0} \rrbracket=-1
$$

Thus, the generalized Poisson bracket is:

$$
\llbracket f, g \rrbracket=\{f, g\}-\left(\frac{\partial f}{\partial x^{0}} \frac{\partial g}{\partial p_{0}}-\frac{\partial f}{\partial p_{0}} \frac{\partial g}{\partial x^{0}}\right)
$$

Such expression has already been derived [62] based on the invariance of the Lagrange brackets which will not be discussed here. The reader will see in the forthcoming two examples that the meaning of the general parametrization $\lambda$ is intimately related to the choice of extended Hamiltonian $\boldsymbol{H}$.

Alternatively one can absorb the "- $"$ into the $\eta_{\mu \nu}$ and thus adopt instead the signature $\{-,+,+,+\}$ for $\eta_{\mu v}$. Then the expression $\sqrt{g(\vec{v}, \vec{v})}$ in Section 3.1 will become $\sqrt{-g(\vec{v}, \vec{v})}$. This is an alternative approach that seems to arrive at very much the same general conclusions [21,22].

\subsection{Implementing the Hamiltonian Constraint}

If one uses the extended Poisson bracket $\llbracket f, g \rrbracket$ one can extend also the classical Hamiltonian $H$ in a suitable way:

$$
H \rightarrow \boldsymbol{H}=H+?
$$

so that the Hamiltonian's evolution equations are recovered:

$$
\frac{d f}{d x^{0}}=\{f, H\}+\frac{\partial f}{\partial x^{0}} \rightarrow \frac{d f}{d \lambda}=\llbracket f, \boldsymbol{H} \rrbracket .
$$

If $\lambda$ is chosen to be the coordinate time $t$, then one has:

$$
\begin{aligned}
\frac{d f}{d t} & =\{f, H\}+\frac{\partial f}{\partial t}=\llbracket f, \boldsymbol{H} \rrbracket \\
\llbracket f, \boldsymbol{H} \rrbracket & =\{f, \boldsymbol{H}\}-\left(\frac{\partial f}{\partial x^{0}} \frac{\partial \boldsymbol{H}}{\partial p_{0}}-\frac{\partial f}{\partial p_{0}} \frac{\partial \boldsymbol{H}}{\partial x^{0}}\right) .
\end{aligned}
$$

To get the usual $\frac{\partial f}{\partial t}$ that is the explicit time derivation of an observable $f$, the extended Hamiltonian $\boldsymbol{H}$ should be chosen to be:

$$
\boldsymbol{H}=H\left(q_{i}, p_{i}\right)-c p_{0}
$$

Then one has:

$$
\begin{aligned}
\frac{d f}{d x^{0}} \rightarrow \frac{d f}{d \lambda} & :=\llbracket f, \boldsymbol{H} \rrbracket=\{f, \boldsymbol{H}\}-\left(\frac{\partial f}{\partial x^{0}} \frac{\partial \boldsymbol{H}}{\partial p_{0}}-\frac{\partial f}{\partial p_{0}} \frac{\partial \boldsymbol{H}}{\partial x^{0}}\right)= \\
& =\{f, H\}-\left(\frac{\partial f}{\partial x^{0}} \frac{\partial\left(-c p_{0}\right)}{\partial p_{0}}-\frac{\partial f}{\partial p_{0}} \frac{\partial H}{\partial x^{0}}\right) \\
& =\{f, H\}+c \frac{\partial f}{\partial x^{0}}+\frac{\partial f}{\partial p_{0}}\left(\frac{\partial H}{\partial x^{0}}\right)
\end{aligned}
$$

This is the usual Hamiltonian evolution equation as long as there is no explicit time dependence in the classical Hamiltonian $H=H\left(q_{i}, p_{i}\right)$, which is often the case. Even if there is an explicit time dependence of the classical Hamiltonian, the extra term will kick-in only if the observable $f$ depends explicitly on $p_{0}$. For example, if $f=p_{0}$ then the extra term results in the correct expression for the rate of change of $p_{0}$, The extended Poisson 
bracket $\llbracket f, g \rrbracket$ and the extended Hamiltonian $\boldsymbol{H}=H\left(q_{i}, p_{i}\right)-c p_{0}$ will recover the standard Hamiltonian formalism. Upon imposing the requirement $\boldsymbol{H}=0$, one can determine the phase-space of the system which will also recover the usual relationship that the classical Hamiltonian $H\left(q_{i}, p_{i}\right)$ is related to the energy of the system $H\left(q_{i}, p_{i}\right)=E=c p_{0}$.

An apparent drawback is that $\boldsymbol{H}=H\left(q_{i}, p_{i}\right)-c p_{0}$ does not seems to be generally covariant. However, this is due to the choice of parametrization $\lambda=x^{0}=c t$ that has been identified with the laboratory time coordinate $t$. In a theory that is built upon a reparametrization-invariant first-order homogeneous Lagrangian with $\boldsymbol{H} \equiv \mathbf{0}$ then $\boldsymbol{H}$ is generally covariant by construction.

In the alternative approach developed in [21,22] that is using $\left\{q^{\mu}, p_{v}\right\}=\delta_{v}^{\mu}$ one arrives at an extended Hamiltonian that has a + sign in front of $p_{0}$, that is, $\boldsymbol{H}=H\left(q_{i}, p_{i}\right)+c p_{0}$. Such expression, in general, does not convey the significance of $H \equiv 0$ because one usually expects the Hamiltonian $H\left(q_{i}, p_{i}\right)$ as well as $p_{0}$ to be related to the energy of a system and for non-interacting systems, all these are expected to be positive. Furthermore, the expression may be confused to stand for the usual addition of various energy components such as energy of a particle $c p_{0}$ and interaction energy with the environment $H\left(q_{i}, p_{i}\right)$. This could be mitigated partially if one uses the dual of $p_{0}$ instead because for signature $\{-,+, \ldots,+\}$ this results in $\boldsymbol{H}=H\left(q_{i}, p_{i}\right)-c p^{0}$.

\subsection{The Schrödinger Equation}

Using the above described extended Hamiltonian formalism, one can look at the corresponding quantum picture. Most of it is already in the correct form:

$$
\begin{aligned}
\{A, B\} & \rightarrow \llbracket A, B \rrbracket \rightarrow \frac{1}{i \hbar}[\hat{A}, \hat{B}] \\
\left\{q_{i}, p_{i}\right\}=1 & \rightarrow \llbracket q_{i}, p_{i} \rrbracket=1 \\
& \Rightarrow[\hat{q}, \hat{p}]=i \hbar \Rightarrow \hat{p}_{i}=-i \hbar \frac{\partial}{\partial q_{i}} \\
\left\{q_{0}, p_{0}\right\}=-1 & \rightarrow \llbracket q_{0}, p_{0} \rrbracket=-1 \\
& \Rightarrow\left[\hat{q}_{0}, \hat{p}_{0}\right]=-i \hbar \Rightarrow \hat{p}_{0}=i \hbar \frac{\partial}{\partial q_{0}} \\
& \mathcal{H}=\{\boldsymbol{H} \psi \equiv 0\}
\end{aligned}
$$

Looking at the expression (41) for $\lambda=q_{0}=c t$, one now has:

$$
c p_{0} \psi=H\left(q_{i}, p_{i}\right) \psi
$$

which is exactly the Schrödinger equation [80]:

$$
i \hbar \frac{\partial \psi}{\partial t}=H\left(q_{i}, p_{i}\right) \psi
$$


One can arrive at the Schrödinger equation even with the alternative choice of the extended Poisson Bracket, and the opposite sign in the expression of $\hat{p}_{0}$ would be handy and collaborate with + sign in the alternative extended Hamiltonian $\left(\boldsymbol{H}=H\left(q_{i}, p_{i}\right)+c p_{0}\right)$ to produce the correct Schrödinger equation [21,22].

The Schrödinger Equation (47) along with its dual equation show that the norm of the state $\psi$, which is a solution to (47), and will be conserved as long as $H\left(q_{i}, p_{i}\right)$ is self-dual operator; that is, $H\left(q_{i}, p_{i}\right)$ is a Hermitian operator $\left(H^{\dagger}\left(q_{i}, p_{i}\right)=H\left(q_{i}, p_{i}\right)\right)$ within the considered Hilbert space with the appropriate inner product. Thus, within the Schrödinger's picture of Quantum Mechanics, the unitary evolution of a state is a well-understood property of the corresponding picture. The Schrödinger's picture of Quantum Mechanics is also equivalent to the Heisenberg's picture based on the Hamiltonian's evolution Equation (40). In the Heisenberg's picture, the state of the system is un-mutable, while the measurement operators are undergoing a unitary evolution according to equation similar to Heisenberg's evolution equations [86]. In this section, the Schrödinger Equation (47) was derived upon the specification of a particular mathematical expression for the extended Hamiltonian $\left(\boldsymbol{H}=H\left(q_{i}, p_{i}\right)-c p_{0}\right)$ that is relevant when the process parametrization $\lambda$ takes upon the meaning of coordinate time $t$ and thus (47) follows from (45) via (46) and (44). Under this specification, the standard Schrödinger's picture is recovered along with all its wellknown unitary evolution and probability interpretation. The Equation (45), that should be used to define the Hilbert space of the corresponding system under consideration, is also form-invariant upon a much larger class of unitary transformations. What will be the form and meaning of the unitary evolution, Schrödinger's or Heisenberg's or some other type, is not clear in general since one has to determine the meaning of the parameter $\lambda$, which is intimately related to the specific mathematical expression that would encode the Hamiltonian constraint (45). The connection between the specific meaning of parameter $\lambda$ and the mathematical form of the extended Hamiltonian $\boldsymbol{H}$ is illustrated in more detail in the following sections as well as in Appendix A. Nevertheless, $\mathcal{H}=\{\boldsymbol{H} \psi \equiv 0\}$ given by (45) is a strong justification for the linear superposition principle that is a key property of the Quantum Mechanics.

\section{The Meaning of $\lambda$ and the Role of the Hamiltonian Constraint}

In this section, we discuss the meaning of the time (evolution) parameter $\lambda$ as related to the choice of expressing the Hamiltonian constraint of a reparametrization-invariant system based on first-order homogeneous Lagrangians in the velocities. Up to our best knowledge, the general functional form of the first-order homogeneous Lagrangians in $n$-dimensional space-time is not fully understood yet $[6,18,27]$. Nevertheless, since any motion of an object can be viewed either in a co-moving frame, where the object is practically at rest and thus moving only through time while all the other coordinates are then irrelevant, or one can employ curvilinear coordinates where the motion is only along one of the spatial coordinates; that is, the motion is along its trajectory coordinate while all other spacial curvilinear coordinates are fixed.

In this respect, the following sections still bear significant physical content and the results are valid in the general context as seen in Appendix A for the case of the relativistic particle; however, we prefer the main exposition below to be without the unnecessary clutter of multi-dimensional notations.

\subsection{The Picture from Lagrangian Mechanics' Point of View}

For the simplest possible case of only one space-time coordinate $q$, one has an explicit unique form for the Lagrangian based on the Euler's equation for homogeneous functions of the first-order in the velocity:

$$
v \frac{\partial L(q, v)}{\partial v}=L(q, v) \Rightarrow L(q, v)=\phi(q) v
$$


The action $\mathcal{A}$ will take a very simple form:

$$
\mathcal{A}=\int L(q, v) d \lambda=\int \phi(q) v d \lambda=\int \phi(q) d q
$$

The Euler-Lagrange equations are now:

$$
\frac{d p}{d \lambda}=\frac{\partial L}{\partial q}, p:=\frac{\partial L}{\partial v}=\phi(q)
$$

The Hamiltonian function is then:

$$
H=p v-L \equiv 0
$$

At this point, there are two choices for the meaning of the coordinate $q$. It could be a spatial coordinate or a time coordinate. For the present exposition, the time-like coordinate is of special interest, but as a warmup and for comparison we first discuss the space-like case.

\subsubsection{The Proper Length Parametrization and the Onset of Quantum Length Scale}

If one chooses to associate $q$ with a position in space then $v$ can be the coordinate velocity if $\lambda=t$. In general, one has $v=d q / d \lambda$ and looking for the $q(\lambda)$ that minimizes $\mathcal{A}$ tells us a trajectory that has a unique value associated to it-the minimum $\Delta$ for the action $\mathcal{A}$ where $q(\lambda)$ satisfies the Euler-Lagrange equations. However, the Equation (50) are now trivially satisfied for any $q(\lambda)$ since $L(q, v)=\phi(q) v$, and $p=\phi(q)$ :

$$
\frac{d \phi(q)}{d \lambda}=\frac{\partial \phi}{\partial q} v \Leftrightarrow \frac{\partial \phi}{\partial q} \frac{d q}{d \lambda}=\frac{\partial \phi}{\partial q} v
$$

This is very similar to the multi-dimensional case discussed in Reference [34]. The Hamiltonian function is not telling us anything new either, it is just bringing us back to the original expression for $L=p v$ with $p=\phi(q)$.

However, based on conservation of the equivalent Lagrangians $L_{(n)}=\left(L_{1}\right)^{n}$ one can impose $\frac{d L_{1}}{d \lambda}=0$ to ensure conservation of $L_{1}$ that gives us an additional equation:

$$
\begin{array}{r}
\frac{d L_{1}}{d \lambda}=0 \Rightarrow \frac{d \phi(q)}{d \lambda} v+\phi(q) \frac{d v}{d \lambda}=0 \\
\Rightarrow \frac{d v}{d \lambda}=-v^{2} \frac{\partial \ln \phi(q)}{\partial q}
\end{array}
$$

The equation for the rate of change of the velocity $v$ is gauge invariant under the general change of parametrization $\lambda \rightarrow \xi$ given by a general function $\lambda(\xi)$ :

$$
\frac{d\left(v^{-1}\right)}{d \lambda}=\frac{\partial \ln \phi(q)}{\partial q} \Leftrightarrow \frac{d\left(w^{-1}\right)}{d \xi}=\frac{\partial \ln \psi(q)}{\partial q}
$$

where $v=\frac{d q}{d \lambda}, w=\frac{d q}{d \xi^{\prime}}$, and $\phi(q)=\psi(q) \lambda^{\prime}(q)$, with $\lambda^{\prime}(q)=\left.\frac{d \lambda}{d \bar{\xi}}\right|_{q}$.

The gauge invariance can be seen either from the expression for the Lagrangian $L$ within the action $\mathcal{A}$ (49) or from the following direct mathematical considerations. 
Consider first how the left-hand side transforms:

$$
\begin{aligned}
\frac{d\left(v^{-1}\right)}{d \lambda} & =\frac{d}{d \lambda}\left(\frac{d \lambda}{d q}\right) \rightarrow \\
& =\frac{d}{d \lambda(\xi)}\left(\frac{d \lambda(\xi)}{d q}\right)=\frac{d}{\lambda^{\prime} d \xi}\left(\frac{\lambda^{\prime} d \xi}{d q}\right)= \\
& =\frac{d}{d \xi}\left(\frac{d \xi}{d q}\right)+\frac{\lambda^{\prime \prime}}{\lambda^{\prime}}\left(\frac{d \xi}{d q}\right) \\
& =\frac{d}{d \xi}\left(w^{-1}\right)+\frac{d \ln \left(\lambda^{\prime}\right)}{d \xi}\left(\frac{d \xi}{d q}\right) \\
& =\frac{d}{d \xi}\left(w^{-1}\right)+\frac{d \ln \left(\frac{d \lambda}{d \xi}\right)}{d q} \\
\Rightarrow & \frac{d\left(v^{-1}\right)}{d \lambda} \rightarrow \frac{d}{d \xi}\left(w^{-1}\right)+\frac{d \ln \left(\lambda^{\prime}\right)}{d q}
\end{aligned}
$$

the right-hand side transforms as follows:

$$
\frac{\partial \ln \phi(q)}{\partial q} \rightarrow \frac{\partial \ln \left(\psi(q) \lambda^{\prime}\right)}{\partial q}=\frac{\partial \ln (\psi(q))}{\partial q}+\frac{\partial \ln \left(\lambda^{\prime}\right)}{\partial q}
$$

Thus, the second $\left(\ln \left(\lambda^{\prime}\right)\right)$ terms would cancel out.

By looking at $\phi(q)$ in the Equation (54) one can see that if $\phi(q)$, which is the momentum $p$, is constant $\left(p=\phi(q)=\phi_{0}\right)$ during the process then $v$ does not change and is conserved along the path $q(\lambda)=v \lambda+q(0)$ and the constant value of the Lagrangian is $\phi_{0} v\left(L=\phi_{0} v\right)$. If one assumes $\lambda=v^{-1} q$ with $v$ an arbitrary non-zero constant then the Equation (54) demands $\phi(q)$, the momentum $p$, to be constant $p=\phi(q)=\phi_{0}$ :

$$
0=\frac{d\left(v^{-1}\right)}{d \lambda}=\frac{\partial \ln \phi(q)}{\partial q}
$$

In this case a new parametrization can be chosen-the proper-length $l$ so that $\left(d l=\phi_{0} d q=\phi_{0} v d \lambda\right)$ and the value of the Lagrangian becomes one $\left(L=\phi_{0} v \rightarrow L=1\right)$ :

$$
\begin{aligned}
\mathcal{A} & =\int L(q, v) d \lambda=\int \phi(q) v d \lambda=\int \phi(q) d q \\
& \Rightarrow \Delta l=\int \phi_{0} d q=\phi_{0} \Delta q=\phi_{0} v \Delta \lambda
\end{aligned}
$$

The proper-length $l$ can be introduced even in the general case of $\phi(q)$ :

$$
\begin{aligned}
d l & =\phi(q) d q=\phi(q) v d \lambda, \\
\Rightarrow \mathcal{A} & =\int L(q, v) d \lambda=\int d l=\Delta l
\end{aligned}
$$

If one chooses the proper length $d l=\phi(q) d q$ as parametrization, then the Lagrangian is explicitly a constant $(L=1)$ and the "velocity" is $w=d q / d l=\frac{1}{\phi(q)}$, but from the form invariant expression of the action integral, $\psi(q) w d l=\phi(q) v d \lambda=\phi(q) d q=d l$ one also has $L=p w$, which requires $p=\psi(q)=\phi(q)$ so that $L=1$. In this case the Euler-Lagrange equations:

$$
\frac{d p}{d l}=\frac{\partial L}{\partial q}, p:=\frac{\partial L}{\partial w}=\psi(q)=\phi(q)
$$


are trivially satisfied as well:

$$
\frac{d \phi(q)}{d l}=\frac{\partial L}{\partial q} \rightarrow \frac{d \phi(q)}{d q} \frac{d q}{d l}=w \frac{\partial \phi(q)}{\partial q} \rightarrow \frac{1}{\phi(q)} \frac{\partial \phi(q)}{\partial q}
$$

If one looks for any other parametrizations, which correspond to constant $L$ and thus satisfy (54) or the equivalent Equation (55), one can conclude that there is a family of parametrizations up to a constant factor $\lambda_{0}$ related to $\lambda_{0} d \lambda=\phi(q) d q$. For this purpose, consider (55) such that $\lambda=\lambda(q)$ since there is no other variable for $\lambda$ to depend on. Then Equation (55) gives us:

$$
\begin{aligned}
\frac{\partial \ln \phi(q)}{\partial q} & =\frac{d\left(v^{-1}\right)}{d \lambda}=\frac{d}{d \lambda}\left(\frac{d \lambda}{d q}\right) \\
& =\left(\frac{d q}{d \lambda}\right) \frac{d}{d q}\left(\frac{d \lambda}{d q}\right)=\frac{d \ln \lambda^{\prime}(q)}{d q} \\
\Rightarrow & \frac{\partial \ln \phi(q)}{\partial q}=\frac{d \ln \lambda^{\prime}(q)}{d q}
\end{aligned}
$$

with the general solution $\lambda^{\prime}(q)=\phi(q) / \lambda_{0} \Rightarrow \lambda_{0} d \lambda=\phi(q) d q$.

The above considerations show that there is always a choice of parametrization that makes the Lagrangian constant. In particular, in the proper-length parametrization when using $d l=\phi(q) d q$ one can make $L=1$. Of course, there is also the question whether $\phi(q)$ is well behaved in order to establish good equivalence between the $l$ value and the $q$ coordinate of the trajectory of the process with $p=\phi(q)$.

Since $p=\phi(q)$ should be related to the momentum of the system, then a process can be considered classical with conserved linear momentum if a value can be associated to the process and it is independent of the observational length scale $\Delta q$ via the following expression:

$$
p(\Delta q):=\langle\phi\rangle_{\Delta q}=\frac{1}{\Delta q} \int_{0}^{\Delta q} \phi(q) d q=\frac{1}{\Delta q} \int_{0}^{\Delta l} d l=\frac{\Delta l}{\Delta q}
$$

The simplest examples for such averaging with observational length scale are the density of a material, the large scale structure of the Universe, or the average speed of a city bus, or train, etc.

If $p(\Delta q)=\phi(q)=$ const, then the coordinate $q$ is proportional to the proper length $l$. In particular, in the center of mass of a system one can expect $p=0$ which will mean that there is no change in $l$ for the process. However, this may also happen due to quantum effects when quantum fluctuations are canceling out beyond a large enough scale $\Delta$. Furthermore, if one studies natural processes at shorter and shorter scales than one may encounter systems where the proper-length is poorly defined due to fluctuations of $\phi(q)$ and the above formula is not applicable because of limitations at very small scales. The observation of such a length scale $\delta$ can signal the onset of quantum phenomenon.

\subsubsection{The Proper Time Parametrization and the Onset of Quantum Time Scale}

Now, let us consider the possibility that the $q$ coordinate is time-like. In what follows, $q$ will be set to be the laboratory time coordinate $t$ and the rate of its change $d t / d \lambda$ will be denoted with $u$ instead of $v_{0}$ but we will not use $E$ nor $p_{0}$ for $p$ which in this case carry the correct meaning of $p$ :

$$
\begin{gathered}
L_{1}(t, u)=\phi(t) u \Rightarrow u:=\frac{d t}{d \lambda}, p:=\frac{\partial L_{1}}{\partial u}=\phi(t), \\
\frac{d p}{d \lambda}=\frac{\partial L_{1}}{\partial t} \Rightarrow \frac{d \phi(t)}{d \lambda}=u \frac{\partial \phi(t)}{\partial t}
\end{gathered}
$$


The corresponding Hamiltonian function is then:

$$
H=p u-L \equiv 0
$$

but one cannot say anything about the rate with which $u$ is changing. The action $\mathcal{A}$ will take the value $\Delta$ for the overall observed motion:

$$
\mathcal{A}=\int L(t, u) d \lambda=\int u \phi(t) d \lambda=\int \phi(t) d t=\Delta
$$

Since the model is reparametrization invariant, one can define a quantity that different observers can deduce from observations and compare- this is the proper-time parametrization $\tau$ :

$$
d \tau=\phi(t) d t
$$

In this parametrization the action $\mathcal{A}$ will take simpler form:

$$
\mathcal{A}=\int L(t, u) d \lambda=\int u \phi(t) d \lambda=\int \phi(t) d t=\int d \tau=\Delta \tau
$$

and different observers will be able to compare different phases of the process and deduce overall scale factor that will allow identical results.

Furthermore, for the equivalent Lagrangians $L_{(n)}=\left(L_{1}\right)^{n}$ there is an explicit time dependence. Thus, the corresponding Hamiltonian functions will not be integrals of the motion. For example, $H_{(2)}=\left(L_{1}\right)^{2}=\phi(t)^{2} u^{2}$. However, the proper-time parametrization will make $L_{1}=1$ or by requiring $\frac{d L_{1}}{d \lambda}=0$ one will arrive again at:

$$
\frac{d L_{1}}{d \lambda}=0 \Rightarrow \frac{d u}{d \lambda}=-u^{2} \frac{d \ln \phi(t)}{d t}
$$

which has the general solutions $\lambda_{0} d \lambda=\phi(t) d t$ as discussed in the previous section.

However, again if $\phi(t)=\phi_{0}$ is a constant then $u=\zeta$ is a constant too; therefore, the rate of change of $t$ and $\lambda$ are proportional. This means that one can choose the unit of the process time $\lambda$ to be the same as the coordinate time $t$ which makes $u=1$. Therefore, the action integral will give us:

$$
\mathcal{A}=\int L(t, u) d \lambda=\int \phi(t) d t=\Delta \tau \rightarrow \phi_{0} \int d t=\phi_{0} \Delta t=\phi_{0} \zeta \Delta \lambda
$$

Alternatively, if one starts with $\lambda=t$, then one has $u=1, L=\phi(t)$, and $p$ should be assumed to be $\phi(t)$ since that was the case for all other choices of parameterization. Then one can consider the proper time $\tau$ as a new choice of parametrization to study the system. In the proper-time parametrization $L=1$, which is explicitly a constant. It seems that for massive particles/systems one can always expect $L$ to be non-zero and thus in the proper-time parametrization to be set to 1 . Since the corresponding momentum $p=p_{0}=\phi(t)$ should be related to the energy of the system, then a process can be considered classical with conserved energy if a quantity (energy) can be associated to the process and it is independent of the observational time interval $\Delta t$ via:

$$
E=E(\Delta t):=\langle\phi\rangle_{\Delta t}=\frac{1}{\Delta t} \int_{0}^{\Delta t} \phi(t) d t=\frac{1}{\Delta t} \int_{0}^{\Delta \tau} d \tau=\frac{\Delta \tau}{\Delta t}
$$

However, if one studies natural processes at shorter and shorter time scales then one may encounter systems where the proper-time is poorly defined due to fluctuations of $\phi(t)$ and the above formula is not applicable because of fluctuations at very small time scales. The observation of such time scale $\delta$ can signal the onset of quantum phenomenon. 


\subsection{The Picture from Hamiltonian Mechanics Point of View}

Consider now the same system but from the Hamiltonian point of view using the extended Poisson bracket. The main relationships in the Lagrangian formulation based on the Lagrangian $L_{1}(t, u)=\phi(t) u$ are $u:=\frac{d t}{d \lambda}, p:=\frac{\partial L_{1}}{\partial u}=\phi(t)$, and $H=p u-L \equiv 0$. If one considers the choice of parametrization $\lambda$ to be the laboratory time coordinate $t$ then one has $u=1, L_{1}(t, u)=\phi(t)$, and $H=p-L \equiv 0$, which is consistent with the general expression $p:=\frac{\partial L_{1}}{\partial u}=\phi(t)$ that holds for general choice of parameterizations. Thus the general constraint would be to make sure that (in what follows we will use $c=1$ units):

$$
p_{0}=\phi(t)
$$

Here we use explicitly the sub-index zero to emphasize that this is to be related to the energy momentum of a system.

\subsubsection{Hamiltonian Constraint for $\lambda$ in Coordinate-Time Role $(\lambda=t)$}

The above expression immediately suggests an extended Hamiltonian in the spirit of $H \rightarrow \boldsymbol{H}=H-p_{0}$ that will have the form:

$$
\boldsymbol{H}=\phi(t)-p_{0}
$$

Now an interesting question is: How the phase-space coordinates evolve and what is the meaning of $\lambda$ for such choice of extended Hamiltonian? To answer this question one looks at the evolution equation for the function $t$ and for $p_{0}$ :

$$
\frac{d t}{d \lambda}=\llbracket t, \boldsymbol{H} \rrbracket \Rightarrow \frac{d t}{d \lambda}=\llbracket t,\left(-p_{0}\right) \rrbracket=1
$$

Thus this immediately tells us that the choice of $\lambda$ is actually the laboratory time coordinate. Now one has to confirm the consistency by looking at the evolution of $p_{0}$ :

$$
\frac{d p_{0}}{d \lambda}=\llbracket p_{0}, \boldsymbol{H} \rrbracket \Rightarrow \frac{d p_{0}}{d \lambda}=\llbracket p_{0}, \phi(t) \rrbracket=\frac{\partial \phi(t)}{\partial t}
$$

Thus, the choice of $\boldsymbol{H}=\phi(t)-p_{0} \equiv 0$ corresponds to $\lambda=t$ indeed.

\subsubsection{Hamiltonian Constraint for $\lambda$ in the Proper-Time Role $(\lambda=\tau)$}

The constraint in Equation (71) has many possible realizations. Another possibility is:

$$
\boldsymbol{H}=1-\frac{p_{0}}{\phi(t)}
$$

What is the meaning of $\lambda$ for this form of $\boldsymbol{H}$ ? Again one looks at the evolution of $t$ and $p_{0}$ :

$$
\frac{d t}{d \lambda}=\llbracket t, \boldsymbol{H} \rrbracket \Rightarrow \frac{d t}{d \lambda}=\frac{1}{\phi(t)} \llbracket t,\left(-p_{0}\right) \rrbracket=\frac{1}{\phi(t)}
$$

Thus, this is the proper time parametrization choice since $d \lambda=\phi(t) d t=d \tau$. Again one checks the consistency by looking at $p_{0}$ :

$$
\begin{aligned}
& \frac{d p_{0}}{d \lambda}=\llbracket p_{0}, \boldsymbol{H} \rrbracket \Rightarrow \frac{d p_{0}}{d \lambda}=-p_{0} \llbracket p_{0}, \frac{1}{\phi(t)} \rrbracket \\
& \Rightarrow-p_{0} \frac{\partial(\phi(t))^{-1}}{\partial t}=-p_{0}\left(\frac{-1}{\phi(t)^{2}}\right) \frac{\partial \phi(t)}{\partial t}
\end{aligned}
$$


Since one has to keep $p_{0}=\phi(t)$ this finally gives the same expression as in the laboratory coordinate time because $d \lambda=\phi(t) d t=d \tau$ :

$$
\frac{d p_{0}}{d \tau}=\frac{1}{\phi(t)} \frac{\partial \phi(t)}{\partial t} \Rightarrow \frac{1}{\phi(t)} \frac{d p_{0}}{d t}=\frac{1}{\phi(t)} \frac{\partial \phi(t)}{\partial t}
$$

\subsubsection{The Quantum Mechanics Picture and the Positivity of the Energy}

If one applies the Canonical Quantization formalism to the extended Hamiltonian framework with the $\boldsymbol{H}$ for the time coordinate parametrization $\lambda=t$, one obtains the standard Schrödinger equation:

$$
\boldsymbol{H}=\phi(t)-p_{0} \rightarrow \hat{\boldsymbol{H}} \psi(t)=0 \Rightarrow i \hbar \frac{\partial \psi}{\partial t}=\phi(t) \psi
$$

where the wave function solutions $\psi(t)$ are given by:

$$
\psi(t)=\frac{1}{\mathcal{N}} \exp \left[-\frac{i}{\hbar}\left(\int_{0}^{t<\delta} \phi(t) d t+p_{0} \int_{\delta}^{t \gg \delta} d t\right)\right]
$$

In the above expression we have tried to emphasize the two possible regimes for $\phi(t)$. In the second part the integral $\int_{\delta}^{t \gg \delta} \phi(t) d t$ is replaced with its corresponding expression containing the value $p_{0}$ defined by (70) for observational window $\Delta t \gg \delta$ when one expects (71) to be valid. When this term is dominant, which is when the coordinate-time interval of the process $\Delta t \gg \delta$ such that the energy $p_{0}=E$ is conserved, as discussed earlier in the Lagrangian formulation of this system (70), then this is the familiar plane wave with normalization factor $\mathcal{N}=1$. However, for fluctuation of $\phi(t)$ at short time scale $\Delta t \lesssim \delta$, which is the first integral in the above expression, that does not show energy conservation for the process, then the wave function is related to the integral of $\phi(t)$ and the normalization $\mathcal{N}$ may now depend on the size of $\delta$ and the structure of the relevant Hilbert space and the second term may not be present when $\delta \rightarrow \infty$. The value of $\delta$ depends on where there is a scale beyond which $p_{0}$ is conserved; therefore, $\delta$ if it exists, it is a system/process specific. It is a standard procedure to choose $\mathcal{N}$ to be a positive real number that guarantees the state wave function to be normalized to 1 for the chosen inner product [86]. Generally, the inner product in the space of solutions that turns it into a Hilbert space could be tricky and may need appropriate extension of the notion of model space beyond the standard Hilbert space framework [87]. Regardless of the particular choice of the inner product, one can always determine $\mathcal{N}>0$ based on the fact that the inner product results in an appropriate norm with measure that is positive $\|\psi\|^{2}=\langle\psi \mid \psi\rangle>0$ due to the general properties of the inner product.

For the current purpose, however, a running average may be useful. In particular, for the plane waves one can use a standard mathematical scalar product where $\Delta$ would be a sufficiently long observational window for the process such that energy is conserved and thus slight variations in the window time duration $\Delta$ are producing consistent results. Having consistent results is an important assumption here and in this sense is related to the details of the chosen inner-product:

$$
\langle\Psi \mid \Phi\rangle_{\Delta}=\frac{1}{\Delta} \int_{t_{0}}^{t_{0}+\Delta} \Psi^{*} \Phi d t
$$

The interpretation of $\Psi^{*} \Psi$ as probability density over the configuration space (extended space-time configuration space) is still valid. For example, by including a spatial volume $V$ integration in (80) one arrives at a well-normalized state in the case of "standard quantum-mechanical" Newtonian-time description of a system where the states of the system are already specially normalized to 1 such that the volume integral satisfies 
$\int_{V} \Psi^{*} \Phi d^{3} x=1$. In this case, one extends trivially the integration to be over $V \times\left[t_{0}, t_{0}+\Delta\right]$ and one can see the need of the factor $\frac{1}{\Delta}$ to complete the normalization of the state to $100 \%$ probability. That is, doing a measurement within and time window $\left[t_{0}, t_{0}+\Delta\right]$ results in observing the particle somewhere in the space $V$ at some moment between $\left[t_{0}, t_{0}+\Delta\right]$ during the measurement. Even more, the above expression is still reasonable even in the case of plane-waves where the usual quantum mechanics has issues in coming up with a wellnormalizable state formulation. In the case of the plane-waves one may have to consider also the extend of the spacial measurement as part of the $\Delta$ factor. Here the time duration $\Delta$ is the (spacetime) window of an experimental measurement process that plays the role of regularization procedure. In some sense, it is related to the resolution of the measurement since any two plane waves with periods $T_{m}$ and $T_{n}$ such that $\left(T_{m} T_{n}\right) /\left|T_{m}-T_{n}\right|<<\Delta$ will be practically orthogonal. For example, consider a Fourier series based on periods $T_{n}=\varepsilon / n$ with $\varepsilon<<\Delta$ then one has that all members of the series form orthonormal basis since $\varepsilon /|m-n|<<\Delta$ as long as one considers $\varepsilon / \Delta \approx 0$. Finally, the specific value $t_{0}$ is more of a place holder for when the measurements where made, but the outcome of identical experiments should not really depend on it as long as all the external conditions have been also $t_{0}$-independent.

In the case of the proper-time parametrization $\lambda=\tau$ one is facing the question of ordering of the operators that can be resolved by the requirement of Hermiticity of the extended Hamiltonian with respect to the usual QM rules:

$$
\boldsymbol{H}=1-\frac{1}{2}\left(\frac{1}{\phi(t)} p_{0}+p_{0} \frac{1}{\phi(t)}\right)
$$

The corresponding Schrödinger like equation now will have an additional term:

$$
\begin{gathered}
\psi(t)-\frac{1}{2}\left(\frac{1}{\phi(t)} \hat{p}_{0} \psi(t)+\hat{p}_{0} \frac{\psi(t)}{\phi(t)}\right)=0 \\
\psi(t)-\frac{i \hbar}{2} \frac{1}{\phi(t)}\left(\frac{\partial \psi}{\partial t}+\phi(t) \frac{\partial}{\partial t}\left(\frac{\psi(t)}{\phi(t)}\right)\right)=0 \\
i \hbar \frac{\partial \psi}{\partial t}=\left[\phi(t)+\frac{i \hbar}{2}\left(\frac{\partial \ln \phi(t)}{\partial t}\right)\right] \psi(t)
\end{gathered}
$$

Therefore, the amplitude of the original plane wave will be modulated now by an additional factor $\varrho(t)$ satisfying:

$$
\frac{\partial \varrho(t)}{\partial t}=\frac{1}{2}\left(\frac{\partial \ln \phi(t)}{\partial t}\right) \varrho(t)
$$

This factor will not disappear for $\Delta t \gg \delta$ when the energy $p_{0}=E$ is conserved. It will have the form $\varrho(t)=\sqrt{\phi(t)}$ and now the wave function will be:

$$
\psi(t)=\frac{1}{\mathcal{N}} \sqrt{\phi(t)} \exp \left[-\frac{i}{\hbar}\left(\int_{0}^{t<\delta} \phi(t) d t+p_{0} \int_{\delta}^{t \gg \delta} d t\right)\right]
$$

The expression (86) shows that the complex conjugated wave function $\psi(t)^{*}$ should, therefore, be viewed as the wave function for the time reversal process of the original process. Notice that $\phi(t)$ is expected to be positive in order to be physical, which guarantees proper causal relationship as indicated by the relationship between the proper-time and the coordinate-time (66) and discussed in Section 3.2. Furthermore, since (84) is a linear equation for the wave function $\psi(t)$, the solution is determined up to an overall scalar factor. It is a standard procedure to choose $\mathcal{N}$ to be a positive real number that guarantees the wave function to be normalized to 1 for the chosen inner product, in the example case considered, the inner product is given by (80). 
Furthermore, for processes when energy conservation is observed the modulating factor modifies the wave function normalization to $\mathcal{N}=\sqrt{p_{0}}$. The result is very interesting since the positivity of the norm now requires positivity of the energy $E=p_{0}>0$ since $\phi(t) \rightarrow p_{0}$. In the rest frame this should correspond to the rest mass of the particle.

$$
\|\psi\|^{2}=\langle\psi \mid \psi\rangle_{\Delta}=\frac{1}{\mathcal{N}^{2} \Delta} \int_{0}^{\Delta \gg \delta} \phi(t) d t \underset{\Delta \rightarrow \infty}{\longrightarrow} \frac{p_{0}}{\mathcal{N}^{2}}>0 .
$$

Since the normalization factor $\mathcal{N}$ is somewhat arbitrary one may choose $N=1$ and keep track of the overall norm of the state $\|\psi\|^{2}$, in this case one has $\|\psi\|^{2}=p_{0}$. Thus, the the positivity of the norm $\|\psi\|^{2}>0$ implies positivity of the energy $E=p_{0}>0$. Notice that somewhat similar result has been obtained in Reference [21], where the quantummechanical probability density has been related to the energy density of the wave-function potential.

By applying the operator $\hat{p}_{0}$ on the wave function (86) and considering the limit $\phi(t) \rightarrow p_{0}>0$, one has:

$$
\hat{p}_{0} \psi(t)=i \hbar \frac{\partial}{\partial t} \psi(t)=\left(\frac{i \hbar}{2} \frac{\partial \ln \phi(t)}{\partial t}+\phi(t)\right) \psi(t) \rightarrow p_{0} \psi(t)
$$

and by doing the same on the complex conjugated function one has:

$$
\hat{p}_{0} \psi(t)^{*}=i \hbar \frac{\partial}{\partial t} \psi(t)^{*}=\left(\frac{i \hbar}{2} \frac{\partial \ln \phi(t)}{\partial t}-\phi(t)\right) \psi(t)^{*} \rightarrow-p_{0} \psi(t)^{*}
$$

Thus, the complex conjugate wave function corresponds to well-normalized but negative energy state that could also be viewed as a time-reversal state with positive energy since $p_{0}>0$ in both cases:

$$
\begin{aligned}
\psi(t)^{*} & =\frac{1}{\mathcal{N}} \sqrt{\phi(t)} \exp \left[+\frac{i}{\hbar}\left(\int_{0}^{t<\delta} \phi(t) d t+p_{0} \int_{\delta}^{t>\delta} d t\right)\right]= \\
& =\frac{1}{\mathcal{N}} \sqrt{\phi(t)} \exp \left[-\frac{i}{\hbar}\left(p_{0} \int_{\infty}^{\delta} d t+\int_{\delta}^{0} \phi(t) d t\right)\right]
\end{aligned}
$$

The first expression (90) above is the complex conjugated function $\psi(t)$ in (86), while the second expression is the time reversal of $\psi(t)$ in (86) as encoded by the order of integration.

In Appendix A, the multi-dimensional case of the relativistic particle has been considered. The results corresponding to the above discussion are given by Equations (A8) and (A9). As seen from the discussion in Appendix A, there is a preferred choice of an inner product that results in a normalized state when the size of measurement window $\Delta_{\tau}$ is chosen to correspond to the proper-time interval for the measurement. In general, however, an observer could define the inner product based on the measurement window $\Delta_{t}$ corresponding to the coordinate-time $t$ in the lab, in this case, the norm of the state is related to the relativistic factor $\gamma=1 / \sqrt{1-v^{2} / c^{2}}$. The relativistic factor $\gamma$ is usually considered to be positive, and it should be positive since according to the result in (A9) it is related to the norm of the state. The relativistic factor can be expressed also in terms of the energy-momentum components and in the particular case of conserved energy-momentum, the positivity of the energy follows since $p^{0}=\gamma \sqrt{-g_{\mu \nu} p^{\mu} p^{\nu}}$.

6.2.4. The Rate of Change along a Coordinate and Normalizability of the Wave Function

The extended canonical Poisson brackets were chosen to result in the Lorentz-invariant bracket that gives us the usual form for the momentum operators in quantum mechanics along with a reasonable new expression for the evolution equation that involves the 
extended Hamiltonian $\boldsymbol{H}$. In analogy to the evolution equation, one can ask what is the meaning of $\lambda$ if one decides to choose $\boldsymbol{H}$ to be any of the linear momentum generators? If one does so, then one sees that $\boldsymbol{H}=p_{1}$ in the evolution equation corresponds to change along the $q_{1}$ coordinate since then $d \lambda=d q_{1}$ (here 1 indicates any of the spatial coordinates):

$$
\frac{d f}{d \lambda}=\llbracket f, \boldsymbol{H} \rrbracket \rightarrow \frac{d f}{d \lambda}=\llbracket f, p_{1} \rrbracket \Rightarrow \frac{d q_{1}}{d \lambda}=\llbracket q_{1}, p_{1} \rrbracket=1
$$

For $f=p_{1}$ this will give us conservation of $p_{1}$ :

$$
\frac{d f}{d \lambda}=\llbracket f, H \rrbracket \rightarrow \frac{d p_{1}}{d \lambda}=\llbracket p_{1}, p_{1} \rrbracket \Rightarrow \frac{d p_{1}}{d \lambda}=0
$$

If one tries to construct the phase space of this system using $H=0$ one will get only $p_{1}=0$ which is expected to correspond to non-moving particle along $q_{1}$. To get to the more accurate expression one must take advantage of the fact that $\boldsymbol{H}$ relevant for the evolution equation is determined up to a constant. That is, $\boldsymbol{H}$ and $\boldsymbol{H}+$ const will give us the same evolution equations. Thus, in the above example it is more relevant to consider $\boldsymbol{H}=p_{1}-p_{1}(0)$. The meaning of the constant becomes clear from the condition $\boldsymbol{H}=0$ on the states to be considered; then the constant is the value of the conserved momentum $p_{1}(0)$. This is in agreement with the discussion on the Lagrangian $L=\phi(q)(d q / d \lambda)$ when $q$ was a spatial coordinate and demanded constant value of $\phi(q)=\phi_{0}=p_{1}(0)$ by the choice of parametrization $q=\lambda+q(0)$. Furthermore, since $\boldsymbol{H}=p_{1}-\phi_{0}$ can also be viewed as equivalent to $\boldsymbol{H}=v\left(p_{1}-\phi_{0}\right)$ when defining the phase space, then this will correspond to $q=$ $v \lambda+q(0)$ parametrization. In this respect, for $\boldsymbol{H}=v\left(p_{1}-p_{1}(0)\right)$ one has the interpretation of the constant $p_{1}(0)$ as $\phi_{0}$ in the Lagrangian formalism and one can see that $L=v \phi_{0}=v p_{1}(0)$.

In this spirit of reasoning, one sees that $p_{0}$ will correspond to backward coordinate time motion when one considers $\boldsymbol{H}=p_{0}-E$, i.e., for $f=t$ one has:

$$
\frac{d f}{d \lambda}=\llbracket f, \boldsymbol{H} \rrbracket \rightarrow \frac{d t}{d \lambda}=\llbracket t, p_{0} \rrbracket \rightarrow \frac{d t}{d \lambda}=-1
$$

and the energy $p_{0}$ is conserved, which is consistent with the choice of a manifold structure determined by $\boldsymbol{H}=p_{0}-E=0$.

From the above discussion, one can conclude that the extended Hamiltonian $\boldsymbol{H}$ can also reflect space transformations back and forth along a spatial coordinate. Therefore, $\boldsymbol{H}$ should be viewed as a generator of transformations along a path $\boldsymbol{H}=0$ reflects the laboratory coordinate expression of the path as viewed by the observer and gives the relationship between the laboratory coordinate $q_{1}$ and the corresponding momentum $p_{1}$ along the space-like path. When the path is a process, thus time-like related curve, then $\boldsymbol{H}$ is the extended Hamiltonian describing the relationship between the time coordinate $t$ and the energy of the process $E=p_{0}$ as seen by the observer.

Going back to the one spatial coordinate case, the corresponding quantum picture now is based on $\boldsymbol{H}=p_{1}-p_{1}(0)$ and gives:

$$
\boldsymbol{H}=p_{1}-\phi(q) \rightarrow \hat{\boldsymbol{H}} \psi(t)=0 \Rightarrow-i \hbar \frac{\partial \psi}{\partial q}=\phi(q) \psi
$$

where the wave function solutions $\psi(q)$ are given by:

$$
\psi(q)=\frac{1}{\mathcal{N}} \exp \left[\frac{i}{\hbar}\left(\int_{0}^{q<\delta} \phi(q) d q+p_{1} \int_{\delta}^{q \gg \delta} d q\right)\right]
$$

For spatial coordinate interval of the process $\Delta q \gg \delta$ such that the momentum $p_{1}$ is conserved, as discussed earlier in the Lagrangian formulation of this system (61), this is the familiar plane wave with normalization factor $\mathcal{N}=1$ if one switches to $q=v t$. 
However, for fluctuation of $\phi(q)$ at short length scale $\Delta q \lesssim \delta$ that does not show momentum conservation for the process then the wave function is related to the integral of $\phi(q)$ and the normalization $\mathcal{N}$ may depend on the size of $\delta$ and the structure of the relevant Hilbert space. Again the inner product in the space of solutions that turns it into a Hilbert space could be tricky, but a running average may be useful. However, for the plane waves one can use the standard inner product where $\Delta$ would be a sufficiently long observational window for the process such that momentum is conserved and thus slight variations in the window size $\Delta$ are producing consistent results for the structure of the Hilbert space:

$$
\langle\Psi \mid \Phi\rangle_{\Delta}=\frac{1}{\Delta} \int \Psi^{*} \Phi d q
$$

Unlike the one-time coordinate case, here one does not have any limitation on the sign of the linear momentum and if one views the process as a moving particle with velocity $v$ then the complex conjugated wave function $\psi(q)^{*}$ would correspond to a particle moving with opposite momentum or equivalently with opposite direction of the velocity. The expression (96) shows that the complex conjugated wave function $\psi(q)^{*}$ should, therefore, be viewed as the wave function for the directionally reversed process of the original process.

One can also construct the extended Hamiltonian $\boldsymbol{H}$ for the proper-length parameterization or motion along a curved path by looking at $\boldsymbol{H}=\left(p_{1} / \phi\left(q_{1}\right)\right)-\chi\left(q_{1}\right)$ which gives us the proper-length relationship $d l=d \lambda=\phi\left(q_{1}\right) d q_{1}$ and $\chi\left(q_{1}\right)$ should be such that the Hamiltonian constraint $\boldsymbol{H}=0$ gives $p_{1}=\phi\left(q_{1}\right)$ as before. Thus, $\chi\left(q_{1}\right)=1$ finally gives us the $\boldsymbol{H}$ for proper-length parameterization:

$$
\begin{gathered}
H=\frac{p_{1}}{\phi\left(q_{1}\right)}-1 \\
\frac{d f}{d \lambda}=\llbracket f, \boldsymbol{H} \rrbracket \rightarrow \frac{d q_{1}}{d \lambda}=\llbracket \llbracket q_{1}, \frac{p_{1}}{\phi\left(q_{1}\right)}-1 \rrbracket \rightarrow \frac{d q_{1}}{d \lambda}=\frac{1}{\phi\left(q_{1}\right)} \llbracket q_{1}, p_{1} \rrbracket=\frac{1}{\phi\left(q_{1}\right)} \\
\frac{d f}{d \lambda}=\llbracket f, \boldsymbol{H} \rrbracket \rightarrow \frac{d p_{1}}{d l}=p_{1} \llbracket p_{1}, \frac{1}{\phi\left(q_{1}\right)} \rrbracket=p_{1} \frac{1}{\phi\left(q_{1}\right)^{2}} \frac{\partial \phi\left(q_{1}\right)}{\partial q_{1}}=\frac{\partial \ln \phi\left(q_{1}\right)}{\partial q_{1}}=\frac{d \phi\left(q_{1}\right)}{d l}
\end{gathered}
$$

This corresponds to the previous result obtained in the Lagrangian formalism.

The corresponding quantum picture now is very similar to the proper-time quantization with additional term in the Schrödinger like equation:

$$
\begin{aligned}
\boldsymbol{H}= & \frac{1}{2}\left(\frac{1}{\phi(q)} p_{1}+p_{1} \frac{1}{\phi(q)}\right)-1 \rightarrow \hat{\boldsymbol{H}} \psi(t)=0 \\
& \frac{1}{2}\left(\frac{1}{\phi(q)} \hat{p}_{1} \psi(q)+\hat{p}_{1} \frac{\psi(q)}{\phi(q)}\right)=\psi(q) \\
- & \frac{i \hbar}{2} \frac{1}{\phi(q)}\left(\frac{\partial \psi}{\partial q}+\phi(q) \frac{\partial}{\partial q}\left(\frac{\psi(q)}{\phi(q)}\right)\right)=\psi(t) \\
- & i \hbar \frac{\partial \psi}{\partial q}=\left[\phi(q)-\frac{i \hbar}{2}\left(\frac{\partial \ln \phi(q)}{\partial q}\right)\right] \psi(q)
\end{aligned}
$$

Therefore, the amplitude of the original wave function will be modulated now by an additional factor $\varrho(q)$ satisfying:

$$
\frac{\partial \varrho(q)}{\partial q}=\frac{1}{2}\left(\frac{\partial \ln \phi(q)}{\partial q}\right) \varrho(q)
$$

This factor, $\varrho(q)=\sqrt{\phi(q)}$, will now become the main part of the wave function if one views the system in the center of mass frame where $p_{1}=0$. 


$$
\psi(q)=\frac{1}{\mathcal{N}} \sqrt{\phi(q)} \exp \left[\frac{i}{\hbar}\left(\int_{0}^{q<\delta} \phi(q) d q+p_{1} \int_{\delta}^{q>\delta} d q\right)\right] \rightarrow \frac{1}{\mathcal{N}} \sqrt{\phi(q)}
$$

In general, the normalizability of the wave-function implies positivity of $p_{1}>0$ as in the previous case of the $p_{0}$ in the proper-time parametrization. However, the directionality is encoded in the sign of the phase factor and whether one is looking at $\psi$ or its complex conjugate $\psi^{*}$. Thus, for conserved non-zero momentum $p_{1}>0$ the normalization becomes $\mathcal{N}^{2}=p_{1}$ :

$$
\|\psi\|^{2}=\langle\psi \mid \psi\rangle_{\Delta}=\frac{1}{\mathcal{N}^{2} \Delta} \int_{0}^{\Delta>>\delta} \phi(q) d q \underset{\Delta \rightarrow \infty}{\longrightarrow} \frac{p_{1}}{\mathcal{N}^{2}} .
$$

However, in the center of mass frame where $p_{1}=0$ this changes the wave-function normalization $\mathcal{N}$ to be related to the details of the quantum fluctuations of $\phi(q)$ since $\delta<\Delta \rightarrow \infty$ leads to:

$$
\|\psi\|^{2}=\langle\psi \mid \psi\rangle_{\Delta}=\frac{1}{\mathcal{N}^{2} \Delta} \int_{0}^{\Delta \gg \delta} \phi(q) d q \underset{\Delta \rightarrow \infty}{\longrightarrow} 0 .
$$

Thus the effects of the quantum phenomenon disappear when the system is viewed at coarse-grain scale $\Delta \gg \delta$. This may indicate that the inner product in the Hilbert space may have to be redefined:

$$
\langle\Psi \mid \Phi\rangle=\int \Psi^{*} \Phi d q
$$

Now the normalizability of the wave function is related to the usual spatial localization of the physically relevant states $\psi$ that was modulated by the factor $\sqrt{\phi}$.

$$
\|\psi\|^{2}=\langle\psi \mid \psi\rangle=\frac{1}{\mathcal{N}^{2}} \int_{0}^{\delta} \phi(q) d q .
$$

\subsubsection{The Notion of Time Reversal}

In the discussion above, we have shown that the meaning of the process time parameterization $\lambda$ is intimately related to the choice of the Hamiltonian constraint $H$ as expressed in the laboratory. Changing $\boldsymbol{H}$ to its negative $\boldsymbol{H} \rightarrow-\boldsymbol{H}$ does not change the phase space determined by the Hamiltonian constraint $\boldsymbol{H}=0$, but changes the choice of parametrization $\lambda$ to $\xi$ that are now time reversal to each other $d \xi=-d \lambda$. One can see this by comparing the evolution equations of the coordinate time $t$ :

$$
\frac{d t}{d \lambda}=\llbracket t, \boldsymbol{H} \rrbracket \rightarrow \frac{d t}{d \xi}=\llbracket t,(-\boldsymbol{H}) \rrbracket=-\frac{d t}{d \lambda}
$$

Thus, if one considers

$$
\boldsymbol{H}=p_{0}-\phi(t)
$$

in the earlier example above, then one would have:

$$
\frac{d t}{d \xi}=\llbracket t, \boldsymbol{H} \rrbracket \rightarrow \frac{d t}{d \xi}=\llbracket t, p_{0}-\phi(t) \rrbracket=-1
$$

from where one can deduce that $d \xi=-d t$. If one observed that the energy $E=p_{0}$ did not change during the process then this will correspond to a time reversal process. For example, if there are two "identical" clocks one in the laboratory and the other outside and one observes and compare the time from both. Then, one can conclude that one clock is running backwards. This way, it will be possible for models based on reparametrization invariance formalism to have time reversal as a symmetry along with the common arrow of time due to the positivity of the energy (the rest mass of the observers). 


\subsection{The Meaning of $\lambda$ and $\boldsymbol{H}$ in the Extended Phase-Space}

From the previous discussion, we understand that the phase-space momentum coordinates $p_{i}$ can be considered as generators of forward motion along the corresponding coordinates $q_{i}$, while the time and energy coordinate stand out in that $p_{0}$ will correspond to backward coordinate time transformation. In a similar way, the extended Hamiltonian $\boldsymbol{H}$ defines the evolution of a system's observables $f$ along a process parametrized by $\lambda$. In the observer's coordinate frame, $\boldsymbol{H}$ defines the relevant phase-space via $\boldsymbol{H}=\mathbf{0}$ along with equations that tell the observer how the process will unfold from one stage (state), determined by a point in the phase-space, to the nearby stage (state)-another point in the phase space. This is different from the Lagrangian formulation where the configuration space $M$ and its co-tangential space TM that contains the coordinates and the velocities have to be "predetermined" in a way that has nothing to do with the Lagrangian $L$. The Lagrangian, however, tells how the process should be embedded in the tangential space $T M$ by using the Euler-Lagrange equations of motion expressed in a specific laboratory coordinate frame. The phase-space, in this case, is determined by the initial conditions and it is expected to be a sub-manifold of TM upon the evolution using the Euler-Lagrange equations. In the laboratory coordinate frame, the choice $\lambda=t$ is the natural first choice for the process parametrization. However, upon investigation of the system in the Lagrangian formulation one may arrive at the notion of a proper-time $\tau$ that may be a more useful choice of parametrization of a process that should be detached from the choice of a laboratory coordinate frame in the sense that this is the unique laboratory frame where all the special velocities are zero and the time-speed $u$ is 1 . That is, in an arbitrary laboratory frame the various momenta are determined from $p_{\mu}=\partial L / \partial v^{\mu}$ and evolve according to Euler-Lagrange equations $d p_{\mu} / d \lambda=\partial L / \partial x^{\mu}$, but there is the unique co-moving frame where $v^{0}=d x^{0} / d \lambda=d t / d \lambda=1$ and $v^{i}=d q^{i} / d \lambda=0$. Then, for homogeneous Lagrangians of first-order the phase space should be determined by an additional requirement such as parallel transport that conserves the norm of the vectors $(d l / d \lambda=0)$.

In the extended Hamiltonian framework, the phase-space is determined from $\boldsymbol{H}=0$ and the evolution of the coordinates and momenta are governed by the evolution equation via the extended Poisson bracket $d f / d \lambda=\llbracket f, \boldsymbol{H} \rrbracket$ the specific choice of $\boldsymbol{H}$ then tells us the details about the coordinate frame where the observer is studying the process. The reparametrization-invariance was explicit in the Lagrangian framework due to the use of first-order homogeneous Lagrangians in the velocities. In the extended Hamiltonian formulation this is somehow encoded in the extended Hamiltonian $\boldsymbol{H}$ and the structure of the phase-space determined from $\boldsymbol{H}=0$. To understand how the extended Hamiltonian should change when one changes the choice of parametrization one can consider the extended Poisson bracket evolution equation for two different parametrizations that are related by $\lambda(\xi)$ :

$$
\frac{d f}{d \lambda}=\llbracket f, \boldsymbol{H}_{\lambda} \rrbracket \rightarrow\left(\frac{d \xi}{d \lambda}\right) \frac{d f}{d \xi}=\llbracket f, \boldsymbol{H}_{\lambda} \rrbracket \rightarrow\left(\frac{d \xi}{d \lambda}\right) \llbracket f, \boldsymbol{H}_{\xi} \rrbracket=\llbracket f, \boldsymbol{H}_{\lambda} \rrbracket
$$

This can be satisfied if $d \xi \boldsymbol{H}_{\xi}=d \lambda \boldsymbol{H}_{\lambda}+d \lambda I$ where $I$ is an integral of the process $(\llbracket I, H \rrbracket=0)$ such that $I=0$ over the phase space determined by $\boldsymbol{H}=0$. To illustrate this let us consider $\boldsymbol{H}_{t}=\phi(t)-p_{0}$ and $\boldsymbol{H}_{\tau}=1-\frac{p_{0}}{\phi(t)}$. From the specific expressions one can see that $\boldsymbol{H}_{t}=\phi(t) \boldsymbol{H}_{\tau}$ thus $d \tau \boldsymbol{H}_{\tau}=d t \boldsymbol{H}_{t}$ and therefore $d \tau=\phi(t) d t$, which is the usual definition of the relationship of the proper-time to the coordinate time.

If one applies this framework to a moving particle with constant velocity $v$ along the spatial coordinate $q$ one has $q=v t+q(0)$ where $t$ is the new parametrization. Therefore, $\boldsymbol{H}_{q}=p_{1}-p_{1}(0)$ should be related to $\boldsymbol{H}_{t}=v \boldsymbol{H}_{q}+v I=v\left(p_{1}-p_{1}(0)\right)+v I$. The question now is what is the integral of motion I? To find it, one should realize that the configuration space now is two-dimensional $(t, q)$ and the phase-space then will also include $\left(p_{0}, p_{1}\right)$. 
Therefore, $I$ can be determined from the evolution of the equation for the coordinate $t$ and from the requirement that $I$ is an integral of motion:

$$
\frac{d f}{d t}=\llbracket f, \boldsymbol{H}_{t} \rrbracket \rightarrow \frac{d t}{d t}=\llbracket t, \boldsymbol{H}_{t} \rrbracket=\llbracket t, v I \rrbracket=1 \Rightarrow I=-\frac{p_{0}}{v}
$$

This way the corresponding general expression for $\boldsymbol{H}_{t}$ becomes:

$$
\boldsymbol{H}_{t}=v\left(p_{1}-p_{1}(0)\right)-\left(p_{0}-p_{0}(0)\right) \rightarrow \vec{v} \cdot \vec{p}-p_{0}+E
$$

Although this is the physically more relevant system to study due to its possibility to include at least one spatial coordinate and the necessary one-time-coordinate within a Minkowski space-time, it is beyond the scope of the paper which is to analyze the simplest reparametrization-invariant one-dimensional systems for physically relevant consequences and to understand the meaning of the reparametrization parameter $\lambda$.

Based on the examples and the discussions above, one can conclude that the role of the reparametrization parameter $\lambda$ is of a placeholder parameter that is to be clarified after a specific choice of the expression for $\boldsymbol{H}$. However, the usual dynamic time-like meaning of $\lambda$ is often associated with the expression for $\boldsymbol{H}$ that defines the whole phase-space or Hilbert space of the system either via $\boldsymbol{H}=\mathbf{0}$ or via the expression for $\hat{\boldsymbol{H}} \boldsymbol{\psi}=\mathbf{0}$.

\section{Conclusions and Discussion}

Following the main motivation on the importance of reparametrization-invariant models, we have studied the meaning and the roles of the parameter $\lambda$ for the simplest reparametrization-invariant system in one-dimension as well as the physically relevant example of the relativistic particle in any dimensions.

In the process, the extended Hamiltonian formulation discussed was a Lorentzinvariant, and in general, which naturally leads to the standard Schrödinger equation from Quantum Mechanics. The superposition principle, which is the bedrock of Quantum Mechanics and is a natural property of the Hilbert space defined via the Hamiltonian constraint $\hat{H} \Psi=0$. From the examples studied, one can conclude that the proper-length and the proper-time are uniquely identified as the parameterizations where the corresponding Lagrangian becomes a constant of motion with its value equal to 1 . In the corresponding extended Hamiltonian formulation, the corresponding extended Hamiltonian $\boldsymbol{H}$ is easily identifiable in the coordinate $t$ parametrization. While we have shown and confirmed the corresponding expression for the extended Hamiltonian $\boldsymbol{H}$ in the proper-time parametrization $\tau$, it is not clear how to identify the functional form of $\boldsymbol{H}$ in more general $n$-dimensional systems.

In the case of the relativistic particle in gravitational field, however, a Hamiltonian that possesses the key properties of non-relativistic system in proper-time parametrization has been studied in Appendix A.

In general, the connection between the explicit form of the extended Hamiltonian $\boldsymbol{H}$ and the meaning of the parameter $\lambda$ has been illustrated clearly. The quantum mechanical equivalent of such systems has been studied and in the coordinate $t$ parametrization, the usual plane wave has been recovered. An interesting result has emerged from the study of the system using the extended Hamiltonian $\boldsymbol{H}$ for the proper-time parametrization. The wave function now is modulated by a field $\phi(t)$ and in the limit of energy conservation on the macroscopic scale, the energy is forced to be positive in order to have a normalizable wave function.

In the case of the relativistic particle in a gravitational field, the field $\phi(t)$ has been identified with the relativistic factor $\gamma^{-1}$, which is connected to the norm of the quantum mechanical state and thus has to be positive. Furthermore, for a weak time-dependent gravitational field, the energy of the particle receives a factor proportional to the rate of change of the gravitational field. 
This implies the positivity of the rest mass when the field fluctuation can be neglected. Similarly, the coordinate distance $q$ recovers the familiar plane wave with conserved momentum at macroscopic scale and in the proper-length parametrization the wave function now is modulated by the field $\phi(q)$, which should have localizable quantum fluctuations in order to be normalizable. The normalizability of the wave function requires positivity of the energy and momentum variables while the directionality is now encoded in the phase factor of the quantum mechanical wave-function $\psi$ and its complex conjugated $\psi^{*}$. Models based on reparametrization-invariance are likely to have time reversal as a symmetry along with the common arrow of time due to the positivity of the rest mass of the particles. The next steps in this study on the reparametrization-invariant models is to follow the above procedures and to apply them to the relativistic particle in a gravitational field, then to extend particles with spin and to compare the results with the Dirac's formalism, as well as to well-known string theory models.

Some alternative directions for research are also related to explorations of the applicability of the extended Hamiltonian framework to the Born Reciprocity and Reciprocal Relativity ([29], Chapter 9), and to seek appropriate non-commutative symplectic algebra ([29], Chapter 1) extension, as well as Quantized Fields á la Clifford ([29], Chapter 23), which is in one of the original research directions $[6,8]$.

Author Contributions: Conceptualization, V.G.G.; validation, V.G.G. and A.M.; formal analysis, V.G.G. and A.M.; writing—original draft preparation, V.G.G.; writing—review and editing, V.G.G. and A.M. All authors have read and agreed to the published version of the manuscript.

Funding: This research received no external funding.

Institutional Review Board Statement: Not applicable.

Informed Consent Statement: Not applicable.

Data Availability Statement: Not applicable.

Acknowledgments: A.M. expresses his gratitude to his wife for her patience and support. V.G. is extremely grateful to his wife and daughters for their understanding and family support during the various stages of the research presented.

Conflicts of Interest: The authors declare no conflict of interest.

\section{Appendix A. The Relativistic Particle}

In this section the above formalism is illustrated for the case of the relativistic spinless point particle in the presence of the electromagnetic and gravitational background fields. For the strictly relativistic formalism and with more details, one can consult [22]. For treatment of particles with spin via the use of singular Lagrangians see [88-91]. The action $a=\int L(x, v) d \lambda$ with (dimensionless) velocity $v^{\mu}=d x^{\mu} / d \lambda$ is reparametrization invariant when the following relativistic particle Lagrangian is considered:

$$
L=q A_{\mu} v^{\mu}-m c^{2} \sqrt{-g_{\mu \nu} v^{\mu} v^{v}}
$$

Notice that in this section we have chosen a different metric signature $(-+++)$ that is more practical since it avoids sign changes when going from covariant to contravariant spacial components. The corresponding Euler-Lagrange equations are:

$$
\begin{gathered}
\pi_{\mu}=\frac{\partial L}{\partial v^{\mu}}=q A_{\mu}+m c^{2} \frac{g_{\mu \nu} v^{v}}{\sqrt{-g_{\mu \nu} v^{\mu} v^{v}}}, \\
\frac{d \pi_{\rho}}{d \lambda}=\frac{\partial L}{\partial x^{\rho}}=q A_{\mu, \rho} v^{\mu}+\frac{m c^{2}}{2} \frac{g_{\mu v, \rho} v^{\mu} v^{v}}{\sqrt{-g_{\mu \nu} v^{\mu} v^{v}}}
\end{gathered}
$$




\section{Appendix A.1. Coordinate-Time Parametrization}

The choice of coordinate-time parametrization $\left(d \lambda=d t, d x^{0}=c d t\right)$ breaks explicitly the reparametrization invariance and results in dimension-full velocity $v^{\mu}=(c, \vec{v})$. In what follows $\vec{v}=(d \vec{x} / d t)$ denotes the usual three-dimensional velocity and $v^{0}=d x^{0} / d t=c$. Furthermore, $v^{2}=\vec{v} \cdot \vec{v}=g_{i j} v^{i} v^{j}$ is considered implicitly $x$-dependent due to the metric $g_{i j}(x)$. Notice that although the metric signature is $(-+++)$ when $g_{00}$ is written explicitly it will be considered to be positive $\left|g_{00}\right|=-g_{00}$.

Appendix A.1.1. Lagrangian Formulation

The Lagrangian and the corresponding Euler-Lagrange Equations are:

$$
\begin{gathered}
L=q c A_{0}+q A_{i} v^{i}-m c^{2} \sqrt{g_{00}-\frac{\vec{v} \cdot \vec{v}}{c^{2}}}=q c A_{0}+q A_{i} v^{i}-m c^{2} \gamma^{-1}, \\
\pi_{i}=\frac{\partial L}{\partial v^{i}}=q A_{i}+m \frac{v_{i}}{\sqrt{g_{00}-\frac{\vec{v} \cdot \vec{v}}{c^{2}}}}=q A_{i}+m \gamma v_{i}, \\
\frac{d \pi_{i}}{d t}=\frac{\partial L}{\partial x^{i}}=q c A_{0, i}+q A_{j, i} v^{j}+m c^{2} \gamma^{-2} \gamma_{, i},
\end{gathered}
$$

by setting $\gamma=c / \sqrt{-g_{\mu \nu} v^{\mu} v^{v}}=1 / \sqrt{g_{00}-\frac{\vec{v} \cdot \vec{v}}{c^{2}}}$ and by using the kinematical momentum $p_{i}=m \gamma v_{i}$ one has:

$$
\frac{d \pi_{i}}{d t}=\frac{d}{d t}\left(q A_{i}+p_{i}\right)=q c A_{0, i}+q A_{j, i} v^{j}+m c^{2} \gamma^{-2} \gamma_{, i} .
$$

Usually, however, one is interested in the rate of change of the kinematic linear momentum $p_{i}$ :

$$
\begin{gathered}
\frac{d p_{i}}{d t}=q c A_{0, i}+q\left(A_{j, i}-A_{i, j}\right) v^{j}-q c A_{i, 0}+m c^{2} \gamma^{-2} \gamma_{, i}, \\
\frac{d p_{i}}{d t}=q c F_{0 i}+q \overrightarrow{e_{i}} \cdot F \cdot \vec{v}+m c^{2} \gamma^{-2} \gamma_{, i}, \\
\frac{d p_{i}}{d t}=q \overrightarrow{e_{i}} \cdot \vec{E}+q \overrightarrow{e_{i}} \cdot \vec{v} \times \vec{B}+m c^{2} \gamma^{-2} \gamma_{, i} .
\end{gathered}
$$

where the relation between the Faraday tensor $F_{\mu \nu}=\partial_{\mu} A_{\nu}-\partial_{\nu} A_{\mu}$ and the electric $E_{i}=c F_{0 i}$ and magnetic fields $B_{i}=\frac{1}{2} \epsilon_{i j k} F^{j k}$ have be utilized.

Furthermore, since $c d \tau=d s=\sqrt{-g_{\mu \nu} d x^{\mu} d x^{v}}$ then $d s / d \lambda=c d \tau / d t$ and thus $\gamma d \tau=d t$ with $\gamma=c / \sqrt{-g_{\mu \nu} v^{\mu} v^{v}}$. Then from the energy-momentum constraint one has that $(m c)^{2}=\left(p^{0}\right)^{2}-\vec{p}^{2}$, which when combined with $p^{2}=m^{2} \gamma^{2} v^{2}$ and $p^{0}=m \gamma c=E / c$, gives $(m c)^{2}=\left(p^{0}\right)^{2}\left(g_{00}-p^{2} /\left(p^{0}\right)^{2}\right)=(m c)^{2} \gamma^{2}\left(g_{00}-p^{2} /\left(p^{0}\right)^{2}\right)$ thus $\gamma=\left(g_{00}-p^{2} /\left(p^{0}\right)^{2}\right)^{-1 / 2}=$ $p^{0} / \sqrt{-g_{\mu \nu} p^{\mu} p^{v}}$ along with $p_{0} / m c=\gamma$, which shows that $p_{0}$ is an integral of the motion whenever $\gamma$ is an integral of the motion. Notice that the energy-momentum constraint, the Lorentz invariance, and the kinematic momentum expression $p_{\mu}=m \gamma v_{\mu}$ are consistently imposed with respect to the coordinate-time parametrization $t$ and the four-velocity is $v_{\mu}=(c, \vec{v})$.

The Hamiltonian function in the tangential space is non-zero since the reparametrization invariance has been removed by the choice of coordinate-time parametrization. Thus, $h(x, v)$ is related to the energy of the system:

$$
\begin{gathered}
h=\pi_{i} v^{i}-L=\left(q A_{i}+m \gamma v_{i}\right) v^{i}-\left(q c A_{0}+q A_{i} v^{i}-m c^{2} \gamma^{-1}\right), \\
h=-q c A_{0}+m \gamma c^{2}\left(v^{2} / c^{2}+\gamma^{-2}\right)=-q c A_{0}+m \gamma c^{2}\left(\left(\frac{v^{2}}{c^{2}}\right)+\left(g_{00}-\frac{v^{2}}{c^{2}}\right)\right),
\end{gathered}
$$




$$
h=-q c A_{0}+m \gamma c^{2} g_{00} .
$$

In the limit of slow motion, this is the usual non-relativistic expression:

$$
h=-q c A_{0}+m c^{2} \sqrt{g_{00}}+\frac{1}{2} \frac{m}{\sqrt{g_{00}}} \vec{v} \cdot \vec{v}=-q c A_{0}+m c^{2}-m U+\frac{p^{2}}{2 m} .
$$

Notice that it contains only electrostatic and gravitational energy terms and, due to the choices made, $g_{00}$ is positive $\left(g_{00}=\left(1-2 U(x) / c^{2}\right)\right)$.

Appendix A.1.2. Hamiltonian Formulation

In general, for the Hamiltonian formulation one has to use the expression of $h(x, \pi)$ in the $\pi$-space by using $v^{i}=p^{i} /(m \gamma)$ and $p_{i}=\pi_{i}-q A_{i}$. The Hamiltonian function of the system is then:

$$
\begin{gathered}
h=\pi_{i} v^{i}-L=\frac{1}{m \gamma} \pi_{i}\left(\pi^{i}-q A^{i}\right)-\left(q c A_{0}+\frac{1}{m \gamma} q A^{i}\left(\pi_{i}-q A_{i}\right)-m c^{2} \gamma^{-1}\right), \\
h=\frac{1}{m \gamma}\left(\pi_{i}-q A_{i}\right)\left(\pi^{i}-q A^{i}\right)-q c A_{0}+m c^{2} \gamma^{-1} .
\end{gathered}
$$

Working with this Hamiltonian may results in an extra factor of two, if one is not taking into account the derivative of the term $m c^{2} \gamma^{-1}$ :

$$
v^{i}=\frac{d x^{i}}{d t}=\left\{x^{i}, h\right\}=\frac{\partial h}{\partial \pi_{i}}=\frac{2}{m \gamma}\left(\pi^{i}-q A^{i}\right)+\left(\frac{1}{m}\left(\pi_{i}-q A_{i}\right)\left(\pi^{i}-q A^{i}\right)+m c^{2}\right) \frac{\partial \gamma^{-1}}{\partial \pi_{i}} .
$$

Upon using $p_{i}=\pi_{i}-q A_{i}$ along with $p_{0}=m \gamma c$ and $\gamma^{-1}=\sqrt{g_{00}-p^{2} /\left(p^{0}\right)^{2}}$, which implies $\frac{\partial \gamma^{-1}}{\partial p_{i}}=-\gamma p^{i} /\left(p^{0}\right)^{2}$, one gets the correct equations by taking into account the non-relativistic limit $m^{2} c^{2} \gg p_{i} p^{i}$ :

$$
\begin{gathered}
v^{i}=\frac{2}{m \gamma} p^{i}-\left(\frac{1}{m} p_{i} p^{i}+m c^{2}\right) \frac{\gamma p^{i}}{\left(p^{0}\right)^{2}}=\frac{2}{m \gamma} p^{i}-\left(\frac{1}{m} p_{i} p^{i}+m c^{2}\right) \frac{\gamma p^{i}}{(m \gamma c)^{2}}, \\
v^{i}=\frac{1}{m \gamma} p^{i}\left[1-\left(\frac{1}{m} p_{i} p^{i}\right) /\left(m c^{2}\right)\right] \approx \frac{1}{m \gamma} p^{i} .
\end{gathered}
$$

While the rate of change of $\pi_{i}$ is given by:

$$
\frac{d \pi_{i}}{d t}=\left\{\pi_{i}, h\right\}=-\frac{\partial h}{\partial x^{i}}=q c A_{0, i}+\frac{1}{m \gamma} p^{j} p^{k} g_{j k, i}+\left(\frac{1}{m} p_{i} p^{i}+m c^{2}\right) \gamma^{-2} \gamma_{, i} .
$$

The relationship $p^{0}=m \gamma c=E / c$ can be reinforced via the extended Hamiltonian approach where $\boldsymbol{H}=0$ for the following $\boldsymbol{H}$ :

$$
\boldsymbol{H}=h-c p^{0} .
$$

However, starting with (A3) it is not clear how to arrive at at $p^{\mu}=m \gamma v^{\mu}$ and $\gamma^{-1}=$ $\sqrt{g_{00}-p^{2} /\left(p^{0}\right)^{2}}$ unless one considers (A1) and requires that the two expressions for $h$ to always be equal, then one will reproduce the energy-momentum constraint:

$$
m c^{2} \gamma g_{00}=\frac{1}{m \gamma} p^{2}+m c^{2} \gamma^{-1} \Rightarrow\left(p^{0}\right)^{2} g_{00}-p^{2}=(m c)^{2} .
$$

Then, by using $p^{0}=m \gamma c=E / c$ and $p^{i}=m \gamma v^{i}$, one can be recover $\gamma$ and so on.

The evolution of $x^{0}$ and $p^{0}$ in the extended Hamiltonian framework will then be:

$$
\frac{d x^{0}}{d \lambda}=\llbracket x^{0}, \boldsymbol{H} \rrbracket=\llbracket x^{0},-c p^{0} \rrbracket=c \llbracket x^{0}, p_{0} \rrbracket=c .
$$


Therefore, because $x^{0}=c t$, in this case one has $d \lambda=d t$; thus, this choice of $\boldsymbol{H}$ corresponds to the coordinate-time parametrization. Finally, since $h$ has no explicit time dependence, then the energy $c p^{0}=E$ is an integral of the motion whenever $\gamma$ is an an integral of the motion and the metric is static:

$$
\frac{d p^{0}}{d \lambda}=\llbracket p^{0}, \boldsymbol{H} \rrbracket=\llbracket p^{0}, h \rrbracket=-\frac{\partial h}{\partial x^{0}}=\left(\frac{1}{m} p^{2}+m c^{2}\right) \gamma^{-2} \gamma, 0-\frac{1}{m \gamma} p^{i} p^{j} g_{i j, 0} .
$$

Quantization can proceed by using either (A2) or (A3) for the non-relativistic Schrodinger equation. Based on the corresponding Schrodinger's equation, as discussed in the main text, the standard Newtonian-time quantum mechanics and its interpretations follows naturally. As discussed earlier, in the coordinate-time parametrization, the Newtonian-time quantum mechanics is trivially consistent with extended Hamiltonian formalism using $\boldsymbol{H}=h-c p^{0}=0$ along with the extended Poisson bracket.

An interesting question is what parametrization, within the extended Hamiltonian formalism, would $\boldsymbol{H}=1-c p_{0} / h$ correspond to, and what is the extended Hamiltonian that corresponds to the proper-time parametrization? Before we consider these options let us go over the proper-time parametrization case in the Lagrangian formulation.

\section{Appendix A.2. Proper-Time Parametrization}

Appendix A.2.1. Lagrangian Formulation

In proper-time parametrization $\left(d \lambda=d \tau=d s / c, u^{\mu}=d x^{\mu} / d \tau, \sqrt{-g_{\mu \nu} u^{\mu} u^{v}}=c\right)$ :

$$
\begin{gathered}
L=q A_{\mu} u^{\mu}-m c \sqrt{-g_{\mu v} u^{\mu} u^{v}} \\
\pi_{\mu}=\frac{\partial L}{\partial u^{\mu}}=q A_{\mu}+m c \frac{g_{\mu v} u^{v}}{\sqrt{-g_{\mu v} u^{\mu} u^{v}}}=q A_{\mu}+m u_{\mu}, \\
\frac{d \pi_{\rho}}{d \lambda}=\frac{\partial L}{\partial x^{\rho}}=q A_{\mu, \rho} u^{\mu}+\frac{m c}{2} \frac{g_{\mu v, \rho} u^{\mu} u^{v}}{\sqrt{-g_{\mu v} u^{\mu} u^{v}}}, \\
\frac{d}{d \lambda}\left(q A_{\rho}+m u_{\rho}\right)=q A_{\mu, \rho} u^{\mu}+\frac{m}{2} g_{\mu v, \rho} u^{\mu} u^{v}, \\
\frac{d p_{\rho}}{d \lambda}=q\left(A_{\mu, \rho}-A_{\rho, \mu}\right) u^{\mu}+\frac{m}{2} g_{\mu v, \rho} u^{\mu} u^{v},
\end{gathered}
$$

Hamiltonian function is identically zero upon the use of the constraint $\left(-g_{\mu \nu} u^{\mu} u^{v}\right)=c^{2}$ :

$$
\begin{gathered}
h=\pi_{\mu} u^{\mu}-L \\
h=\left(q A_{\mu}+m u_{\mu}\right) u^{\mu}-q A_{\mu} u^{\mu}+m c^{2} \equiv 0
\end{gathered}
$$

Appendix A.2.2. Hamiltonian Formulation

Hamiltonian function using the generalized momentum variables $\pi$ :

$$
h=\pi_{\mu} u^{\mu}-q A_{\mu} u^{\mu}+m c^{2}=\left(\pi_{\mu}-q A_{\mu}\right) u^{\mu}+m c^{2}=\frac{1}{m}\left(\pi_{\mu}-q A_{\mu}\right)\left(\pi^{\mu}-q A^{\mu}\right)+m c^{2}
$$

In this form of the Hamiltonian function $h$ (requiring the zero value as in the TM space form $h(x, v) \equiv 0)$ implies the energy-momentum constraint for the chosen metric signature:

$$
\begin{gathered}
h=\frac{1}{m} p_{\mu} p^{\mu}+m c^{2}, \\
u^{\mu}=\left(\pi^{\mu}-q A^{\mu}\right) / m \Rightarrow p^{\mu}=m u^{\mu} \Rightarrow p_{\mu} p^{\mu}=-m^{2} c^{2}
\end{gathered}
$$


This effectively results in the Klein-Gordon equation $-g_{\mu \nu} p^{\mu} p^{v}+m^{2} c^{2}=0$. However, the corresponding equations of motion produce an extra factor of two due to quadratic dependence of $h$ on the momentum variables:

$$
u^{\rho}=\frac{d x^{\rho}}{d \lambda}=\left\{x^{\rho}, h\right\}=\frac{\partial x^{\rho}}{\partial x^{\mu}} \frac{\partial h}{\partial \pi_{\mu}}=\frac{\partial h}{\partial \pi_{\rho}}=\frac{2}{m}\left(\pi^{\rho}-q A^{\rho}\right),
$$

upon using the result $m u^{\rho}=2\left(\pi^{\rho}-q A^{\rho}\right)$ in the next calculations the term quadratic in the velocity gets a factor of $m / 4$ instead of $m / 2$ as in the Euler-Lagrange equations:

$$
\begin{gathered}
\frac{d \pi_{\rho}}{d \lambda}=\left\{\pi_{\rho}, h\right\}=-\frac{\partial h}{\partial x^{\rho}}=\frac{q}{m} A_{\mu, \rho}\left(\pi^{\mu}-q A^{\mu}\right)+\frac{q}{m}\left(\pi_{\mu}-q A_{\mu}\right) A_{, \rho}^{\mu}+\frac{m}{4} g_{\mu v, \rho} u^{\mu} u^{v}, \\
\frac{d \pi_{\rho}}{d \lambda}=\frac{q}{2} A_{\mu, \rho} u^{\mu}+\frac{q}{2} u_{\mu} A_{, \rho}^{\mu}+\frac{m}{4} g_{\mu v, \rho} u^{\mu} u^{v}=q\left(u^{\mu} A_{\mu}\right)_{, \rho}+\frac{m}{4} g_{\mu v, \rho} u^{\mu} u^{v} .
\end{gathered}
$$

The issue can be resolved by using a modified Hamiltonian $\widetilde{h}=h / 2$, which is justified since $h=0$ anyway:

$$
\widetilde{h}=\frac{1}{2 m}\left(\pi_{\mu}-q A_{\mu}\right)\left(\pi^{\mu}-q A^{\mu}\right)+\frac{m}{2} c^{2} .
$$

The corresponding equations of motion now match well the Lagrangian formulation:

$$
\begin{gathered}
u^{\rho}=\frac{d x^{\rho}}{d \lambda}=\left\{x^{\rho}, \widetilde{h}\right\}=\frac{\partial \widetilde{h}}{\partial \pi_{\rho}}=\frac{1}{m}\left(\pi^{\rho}-q A^{\rho}\right), \\
\frac{d \pi_{\rho}}{d \lambda}=\left\{\pi_{\rho}, \widetilde{h}\right\}=-\frac{\partial \widetilde{h}}{\partial x^{\rho}}=\frac{q}{2} A_{\mu, \rho} u^{\mu}+\frac{q}{2} u_{\mu} A_{, \rho}^{\mu}+\frac{m}{2} g_{\mu v, \rho} u^{\mu} u^{v}=\left(q A_{\mu} u^{\mu}\right)_{, \rho}+\frac{m}{2} g_{\mu v, \rho} u^{\mu} u^{v} .
\end{gathered}
$$

By looking at $d x^{0} / d \lambda$, within the expression above, one see that $m c d t=\left(\pi^{0}-q A^{0}\right) d \lambda$, which is not quite the expression expected in the proper-time parametrization gauge where one should have had $c d \tau=d \lambda \sqrt{-g_{\mu v} u^{\mu} u^{v}}=c d t \sqrt{-g_{\mu \nu} v^{\mu} v^{v}}$. Furthermore, from the expression $d x^{0} / d \lambda$ one also has $c d t / d \lambda=u^{0}=p^{0} / m=E /(m c)$, which should be related to $\gamma$, so one can check if $p^{0}$ is a constant of the motion since $E$ is expected to be:

$$
\begin{aligned}
& \frac{d p^{0}}{d \lambda}=\frac{d\left(\pi^{0}-q A^{0}\right)}{d \lambda}=\left\{\pi^{0}-q A^{0}, \widetilde{h}\right\}=\left\{\pi^{0}, \widetilde{h}\right\}-q\left\{A^{0}, \widetilde{h}\right\}=\frac{d \pi^{0}}{d \lambda}-q \frac{\partial A^{0}}{\partial x^{\mu}} \frac{\partial \widetilde{h}}{\partial \pi_{\mu}}, \\
& \frac{d p^{0}}{d \lambda}=\frac{d \pi_{\mu} g^{\mu 0}}{d \lambda}-q \frac{\partial A^{0}}{\partial x^{\mu}} u^{\mu}=\pi_{\mu} \frac{d g^{\mu 0}}{d \lambda}+g^{\rho 0}\left[\left(q A_{\mu} u^{\mu}\right)_{, \rho}+\frac{m}{2} g_{\mu v, \rho} u^{\mu} u^{\nu}\right]-q \frac{d A^{0}}{d \lambda} .
\end{aligned}
$$

The above expression is quite complicated and not obviously zero, unless the metric tensor is the Lorentz metric $\left(g_{\mu \nu}=\eta_{\mu v}\right)$ for a particle with a zero charge and if the solutions are considered to be the geodesic lines with geodesically transported metric. Thus, in the presence of general gravitational and electromagnetic fields, it may not correspond to an integral of the motion as expected for the energy of a system. The Hamiltonian $\widetilde{h}$ reproduces the corresponding Euler-Lagrange equations derived in proper-time parametrization $(d \lambda=d \tau=d s / c)$ in the Lagrangian formalism, guarantees the mass-shell condition $\left(-g_{\mu \nu} p^{\mu} p^{v}+m^{2} c^{2}=0\right)$ for solutions satisfying $\boldsymbol{H}=\widetilde{h}=0$, and in this respect is related to the Klein-Gordon equation upon quantization; however, it is not clear if this Hamiltonian formulation is really in the proper-time parametrization of the co-moving frame of the particle since $c d t / d \lambda=\left(\pi^{0}-q A^{0}\right) / m=p^{0} / m$ is not connected to $\gamma$ in a clear way.

In the co-moving frame of the particle one would expect $u^{\mu}=(c, 0,0,0)$ and therefore $\pi^{\rho}=q A^{\rho}+m c \delta_{0}^{\rho}$, which gives:

$$
\frac{d \pi_{\rho}}{d \tau}=q \frac{d A_{\rho}}{d \tau}=q A_{\rho, \mu} u^{\mu}=q c A_{\rho, 0},
$$


while the general equations of motion for $d \pi_{\rho} / d \tau$ results in:

$$
\frac{d \pi_{\rho}}{d \tau}=\left(q c A_{0}\right)_{, \rho}+\frac{m}{2} c^{2} g_{00, \rho}
$$

This gives a relationship that is not guaranteed in general since gravity and electromagnetism are independent fields:

$$
\Rightarrow q A_{\rho, 0}=q A_{0, \rho}+\frac{m c}{2} g_{00, \rho} .
$$

However, given that $\sqrt{-g_{\mu \nu} u^{\mu} u^{v}}=c$, then one concludes, using the co-moving frame of the particle where $u^{\mu}=(c, 0,0,0)$, that $g_{\mu=0, v=0}=-1$ and therefore $g_{00, p}=0$. Furthermore, in this case the earlier expression for $d p^{0} / d \tau$ shows that $p^{0}$ is constant of the motion since it is equal to $m c$ due to $\pi^{\rho}=q A^{\rho}+m c \delta_{0}^{\rho}$ :

$$
\frac{d p^{0}}{d \tau}=\frac{d \pi^{0}}{d \tau}-q \frac{\partial A^{0}}{\partial x^{\mu}} \frac{\partial \widetilde{h}}{\partial \pi_{\mu}}=\frac{d \pi^{0}}{d \tau}-q \frac{\partial A^{0}}{\partial x^{\mu}} u^{\mu}=\frac{d \pi^{0}}{d \tau}-q \frac{d A^{0}}{d \tau}=\frac{d(m c)}{d \tau}=0 .
$$

The quantum picture in this case (in the co-moving frame and based on the KleinGordon equation) becomes trivial in the sense that $p^{\mu}=(E / c, 0,0,0)$; thus, the state of the system is represented by a wave function $\Psi=\exp \left(-\frac{i}{\hbar_{c}} t E\right)$ such that $\hat{P}^{\mu} \Psi=p^{\mu} \Psi$ where $\hat{P}^{i}=-i \hbar \partial^{i}$ and $c \hat{P}^{0}=-i \hbar c \partial^{0}$, or equivalently $c \hat{P}^{0}=i \hbar \partial_{t}$ since $x^{0}=c t$. Thus, $\hat{P}^{0} \Psi=p^{0} \Psi$ with $p^{0}=E / c$. However, this corresponds to the coordinate-time parametrization of a co-moving observer seeing a plane-wave, which is not that much about the proper-time parametrization of the process itself.

\section{Appendix A.3. Extended Hamiltonian Framework}

Appendix A.3.1. Proper-Time Parametrization

To have proper-time parametrization in the Extended Hamiltonian formulation one needs to have $d s=c d \tau=d \lambda \sqrt{-g_{\mu \nu} u^{\mu} u^{v}}=c d t \sqrt{-g_{\mu \nu} v^{\mu} v^{v}}=c \gamma^{-1} d t$, by looking at $h$ in (A3), by taking $q=0$ for simplicity since then $\pi_{\mu}=p_{\mu}$, then one can consider the following extended Hamiltonian that provides effectively the same Hamiltonian constraint $\boldsymbol{H}=h+p_{0} c=0$ as before (A4):

$$
\boldsymbol{H}=\left(h+p_{0} c\right) \gamma=h_{c l}+p_{0} c \gamma=\left(\frac{1}{2 m} p_{i} p^{i}+m c^{2}\right)+p_{0} c \gamma
$$

This expression reduces to $p^{0}=m c$ upon $\boldsymbol{H}=0$ at $\vec{p}=0$, then upon $\boldsymbol{H}=0$ the non-relativistic energy follows as well since $p_{0}=-p^{0}$ and in the co-moving frame $\gamma \approx 1$. The extended Hamiltonian formalism then shows that $\lambda$ is the proper time parametrization upon keeping $\gamma$ constant $(\gamma \approx 1)$ :

$$
\frac{d x^{0}}{d \lambda}=\llbracket x^{0}, \boldsymbol{H} \rrbracket=\frac{\partial x^{0}}{\partial x^{\mu}} \frac{\partial \boldsymbol{H}}{\partial p_{\mu}}=\frac{\partial \boldsymbol{H}}{\partial p_{0}}=c \gamma+p_{0} c \frac{\partial \gamma}{\partial p_{0}} \approx c \gamma \Rightarrow \gamma d \lambda=d t .
$$

In this case, $p_{0}$ is an integral of the motion for static spatial metric:

$$
\frac{d p_{0}}{d \lambda}=\llbracket p_{0}, \boldsymbol{H} \rrbracket=-\frac{\partial p_{0}}{\partial p_{\mu}} \frac{\partial \boldsymbol{H}}{\partial x^{\mu}}=-\frac{\partial \boldsymbol{H}}{\partial x^{0}}=\frac{1}{2 m} p^{i} p^{j}\left(-g_{i j, 0}\right)-p_{0} c \gamma, 0 \approx \frac{1}{2 m} p^{i} p^{j}\left(-g_{i j, 0}\right) .
$$

As expected in the instantaneous co-moving frame, the equations of motion correspond to those generated by the classic non-relativistic Hamiltonian $h_{c l}=p^{i} p_{i} /(2 m)+m c^{2}$ since $\gamma \approx 1$ is kept constant.

$$
\frac{d x^{i}}{d \lambda}=\llbracket x^{i}, \boldsymbol{H} \rrbracket=\left\{x^{i}, h_{c l}\right\}+c p_{0}\left\{x^{i}, \gamma\right\} \approx\left\{x^{i}, h_{c l}\right\}
$$




$$
\frac{d p_{i}}{d \lambda}=\llbracket p_{i}, \boldsymbol{H} \rrbracket=\left\{p_{i}, h_{c l}\right\}+c p_{0}\left\{p_{i}, \gamma\right\} \approx\left\{p_{i}, h_{c l}\right\}
$$

Appendix A.3.2. Canonical Quantization

Upon applying the canonical quantization the wave equation based on the extended Hamiltonian $\boldsymbol{H}$ (A6) becomes:

$$
\begin{gathered}
\boldsymbol{H}=\frac{1}{2 m} p_{i} p^{i}+m c^{2}+p_{0} c \gamma=0 \Rightarrow\left[\frac{1}{2 m}(\hat{\vec{P}} \cdot \hat{\vec{P}})+m c^{2}\right] \psi=\gamma c \hat{P^{0}} \Psi=i \hbar \gamma \partial_{t} \psi=i \hbar \partial_{\tau} \psi, \\
i \hbar \partial_{\tau} \psi=\left[-\frac{\hbar^{2}}{2 m}(\vec{\nabla} \cdot \vec{\nabla})+m c^{2}\right] \psi
\end{gathered}
$$

This is the familiar non-relativistic Schrodinger equation when the coordinate-time is also the proper-time of the system. In particular, in the rest frame it implies $\psi \propto \exp \left(-\frac{i\left(K E+m c^{2}\right)}{\hbar} \tau\right)$ up to an overall constant factor due to the linear nature of the equation. Notice that in the above expression the relativistic factor $\gamma$ has been used to switch from the coordinate-time differentiation $\partial_{t}$ to the proper-time differentiation $\partial_{\tau}$.

In the presence of a non-trivial gravitational field the equation in the instantaneous rest frame $(\vec{p}=0)$ becomes:

$$
\boldsymbol{H}=m c^{2}-c p^{0} \gamma=0 \Rightarrow \boldsymbol{H}=1-\frac{p^{0}}{\phi}=0 .
$$

Notice that this equation is the same expression as Equation (75) discussed in main text. Here, we continue by considering fluctuations in the field $\phi$, which is expected to be positive since $m$ and $\gamma$ are usually positive:

$$
\phi=\|\phi(t)\|=\left(\frac{\gamma}{m c}\right)^{-1}=m c \sqrt{g_{00}}=\sqrt{-g_{\mu \nu} p^{\mu} p^{v}},
$$

upon canonical quantization after symmetrization of the Hamiltonian constraint and by dividing with $m c^{2}$ the equation becomes:

$$
\boldsymbol{H}=m c^{2}-\frac{1}{2}\left(p^{0} c \gamma+c \gamma p^{0}\right)=0 \Rightarrow \hat{\boldsymbol{H}}=1-\frac{1}{2}\left(\frac{1}{c \phi} c \hat{P}^{0}+c \hat{P}^{0} \frac{1}{c \phi}\right)=0 .
$$

The quantum mechanical wave function $\psi$ is then given by (see the main text related to Equation (86)):

$$
\psi(t)=\frac{1}{\mathcal{N}} \sqrt{\phi(t)} \exp \left[-\frac{i c}{\hbar} \int \phi(t) d t\right] .
$$

Now by employing (A7), the expression becomes:

$$
\psi(t)=\frac{1}{\mathcal{N}} \sqrt{\phi(t)} \exp \left[-\frac{i}{\hbar} m c^{2} \int_{t_{0}}^{t} \gamma^{-1} d t\right]=\frac{1}{\mathcal{N}} \sqrt{\phi(t)} \exp \left[-\frac{i}{\hbar} m c^{2}\left(\tau(t)-\tau_{0}\right)\right] .
$$

This shows that this state represents a system with conserved quantity (see Equation (70)):

$$
p^{0}=\langle\phi\rangle_{\Delta}=\frac{1}{\Delta} \int_{0}^{\Delta} \phi(t) d t=m c \frac{1}{\Delta} \int_{0}^{\Delta} \gamma^{-1} d t=m c .
$$

By applying $c \hat{P}^{0}$ on the state $\psi$, one concludes that the energy of the particle in the presence of time-fluctuating gravitational field receives an extra contribution due to the gravitational field: 


$$
\begin{aligned}
c \hat{P^{0}} \psi & =i \hbar \partial_{\tau} \psi=i \hbar \gamma \partial_{t} \psi=\left(m c^{2}+i \hbar \gamma \frac{1}{\sqrt{\phi(t)}} \partial_{t} \sqrt{\phi(t)}\right) \psi= \\
& =\left(m c^{2}-\frac{i \hbar}{2} \partial_{\tau} \ln (\gamma)\right) \psi \approx\left(m c^{2}+\frac{i \hbar}{2} \frac{1}{\sqrt{g_{00}}} \partial_{t} \ln \left(g_{00}\right)\right) \psi
\end{aligned}
$$

For weak gravitational fields when $g_{00}=\left(1-2 U(x) / c^{2}\right)$ this results in the following expression:

$$
c \hat{P}^{0} \psi=i \hbar \partial_{\tau} \psi=i \hbar \gamma \partial_{t} \psi=\left(m c^{2}-\frac{i \hbar}{c^{2}} \partial_{t} U\right) \psi .
$$

Thus, as long as the fluctuations in the local gravitational potential $U$ are much smaller than the rest mass of the particle $m c^{4} \gg\left|\hbar \partial_{t} U\right|$ then one can expect conservation of the energy that is matching the rest mass of the particle $E=c p^{0}=m c^{2}$, which is consistent within the Hamiltonian constraint $\boldsymbol{H}=m c^{2}-c p^{0} \gamma=0$ in the rest frame $(\gamma \approx 1)$.

Notice that the norm of the state $\psi$ could be set to 1 if the inner product is defined as:

$$
\langle\psi, \psi\rangle_{\Delta}=\frac{1}{\Delta} \int d t \psi^{*} \psi=\frac{1}{\Delta} \int \frac{\phi(t)}{\mathcal{N}^{2}} d t=\frac{1}{\mathcal{N}^{2} \Delta} \int m c \gamma^{-1} d t=\frac{1}{\Delta} \int d \tau=1,
$$

where $\Delta$ is the measurement window with respect to the rest-frame of the process, thus using proper-time, and the normalization factor $\mathcal{N}^{2}$ is set to be equal to $p_{0}$, that is $\mathcal{N}^{2}=p_{0}=m c$.

Alternatively, if $\Delta$ is the measurement window with respect to the laboratory coordinatetime then the norm of the state $\|\psi\|^{2}$ will correspond to an effective constant factor $\gamma^{-1}$ :

$$
\|\psi\|^{2}=\langle\psi, \psi\rangle_{\Delta}=\frac{1}{\Delta t} \int \psi^{*} \psi d t=\frac{1}{\mathcal{N}^{2} \Delta t} \int m c \gamma^{-1} d t=\frac{m c}{\mathcal{N}^{2}} \frac{\Delta \tau}{\Delta t}=\frac{m c}{\mathcal{N}^{2}} \gamma^{-1}>0 .
$$

In this respect, the positive norm of the wave-function $\left(\|\psi\|^{2}>0\right)$ and the common arrow of time imply $m>0$ and $\gamma>0$ and vice versa. Thus, in order to have a well defined positive norm it is necessary to have $\gamma>0$, which then implies positive $p^{0}>0$ since $p^{0}=\gamma \sqrt{-g_{\mu \nu} p^{\mu} p^{\nu}}$. This implies positivity of the energy $E=c p^{0}>0$ and positivity of the mass as well since $\sqrt{-g_{\mu \nu} p^{\mu} p^{v}}=m c$. In principle one can consider $m<0$ along with $p^{0}<0$, which will still guarantee $\gamma>0$. However, coexistence of $m>0$ along with $m<0$ leads to the proper-time paradox discussed in the section on the arrow of time. Due to the structure of the state $\psi \propto \gamma^{-1 / 2} \exp \left(-\frac{i m c^{2}}{\hbar} \tau\right)$ one can view $\psi^{*}$ as a state corresponding to $m<0$ but it is better to be viewed as anti-particle with $m>0$ that represents the time-reversal state of the original process $\psi$ in this case, the proper-time paradox corresponds to particle-anti-particle annihilation which results in a photon where the proper-time is ill-defined. Furthermore, the above expression shows the importance of the positive mass to guarantee common arrow of time, that is, $\frac{\Delta \tau}{\Delta t}>0$.

\section{References}

1. Anderson, E. The Problem of Time: Quantum Mechanics Versus General Relativity; Fundamental Theories of Physics; Springer International Publishing AG: Cham, Switzerland 2017.

2. Goldstein, H. Classical Mechanics; Addison-Wesley Series in Physics; Addison-Wesley Publishing Company: Boston, MA, USA, 1980; pp. 1-672.

3. Mattingly, D. Modern Tests of Lorentz Invariance. Living Rev. Relativ. 2005, 8, 5. [CrossRef]

4. Pauli, W. Theory of Relativity; Pergamon Press: New York, NY, USA, 1958; pp. 1-272.

5. Gryb, S. A definition of background independence. Class. Quantum Gravity 2010, 27, 215018. [CrossRef]

6. Gueorguiev, V.G. Matter, Fields, and Reparametrization-Invariant Systems. In Geometry, Integrability \& Quantization; Mladenov, I.M., Naber, G.L., Eds.; Coral Press: Sofia, Bulgaria, 2003; Volume IV, pp. 168-177.

7. Gueorguiev, V.G., The Relativistic Particle and its d-brane Cousins. In Proceedings of the 1st Advanced Research Workshop on Gravity, Astrophysics, and Strings at the Black Sea (GAS@BS 2002), Kiten, Bulgaria, 10-16 June 2002; Fiziev, P.P., Todorov, M.D., Eds.; St. Kliment Ohridski University Press: Sofia, Bulgaria, 2003; pp. 148-158. 
8. Gueorguiev, V.G. Aspects of Diffeomorphism Invariant Theory of Extended Objects. In Quantum Theory and Symmetries; Argyres, P.C., Hodges, T.J., Mansouri, F., Scanio, J.J., Suranyi, P., Wijewardhana, L.C.R., Eds.; World Scientific: Singapore, 2004; pp. 234-242. [CrossRef]

9. Gueorguiev, V.G.; Maeder, A. Geometric Justification of the Fundamental Interaction Fields for the Classical Long-Range Forces. Symmetry 2021, 13, 379. [CrossRef]

10. Gueorguiev, V.G. Aspects of Diffeomorphism Invariant Theory of Extended Objects I: The Relativistic Particle and its d-brane Cousins. arXiv 2005, arXiv:math-ph/0512082.

11. Borštnik, M.N.; Nielsen, H.B. Why odd-space and odd-time dimensions in even-dimensional spaces? Phys. Lett. B 2000, 486, 314-321. [CrossRef]

12. van Dam, H.; Ng, Y.J. Why /3+1 metric rather than /4+0 or /2+2? Phys. Lett. B 2001, 520, 159-162. [CrossRef]

13. Saçlioglu, C. Fake $\mathrm{R}^{4}$ s, Einstein spaces and Seiberg-Witten monopole equations. Class. Quantum Gravity 2001, 18, 3287-3292. [CrossRef]

14. Kilmister, C.W. Lagrangian Dynamics: An Introduction for Students; Plenum Press: New York, NY, USA, 1967; pp. 1-136.

15. Cariñena, J.F.; Ibort, L.A.; Marmo, G.; Stern, A. The Feynman problem and the inverse problem for Poisson dynamics. Phys. Rep. 1995, 263, 153-212. [CrossRef]

16. Leighton, R.; Sands, M.; Feynman, R. Feynman Lectures on Physics; Addison-Wesley: Boston, MA, USA, 1965.

17. Gerjuoy, E.; Rau, A.R.P.; Spruch, L. A unified formulation of the construction of variational principles. Rev. Mod. Phys. 1983, 55, 725-774. [CrossRef]

18. Rivas, M. Generalized Lagrangians and spinning particles. arXiv 2001, arXiv:physics/0106023.

19. Dirac, P.A.M. The Theory of Gravitation in Hamiltonian Form. Proc. R. Soc. Lond. Ser. A 1958, 246, 333-343.

20. Gràcia, X.; Pons, J.M. Singular Lagrangians: some geometric structures along the Legendre map. J. Phys. A Math. Gen. 2001, 34, 3047-3070. [CrossRef]

21. Deriglazov, A.; Rizzuti, B.F. Reparametrization-invariant formulation of classical mechanics and the Schrödinger equation. Am. J. Phys. 2011, 79, 882-885. [CrossRef]

22. Deriglazov, A. Classical Mechanics: Hamiltonian and Lagrangian Formalism, 2nd ed.; Springer: Cham, Switzerland, 2016.

23. Klimenko, S.; Nikitin, I. Non-Critical String Theory: Classical and Quantum Aspects; Nova Science: New York, NY, USA, 2007.

24. Lawrie, I.D.; Epp, R.J. Interpretation of time-reparametrization-invariant quantum mechanics: An exactly soluble model. Phys. Rev. D 1996, 53, 7336-7344. [CrossRef] [PubMed]

25. Gryb, S.; Thébault, K. Schrödinger Evolution for the Universe: Reparametrization. Class. Quantum Gravity 2016, $33,065004$. [CrossRef]

26. Kleinert, H. Path collapse in Feynman formula. Stable path integral formula from local time reparametrization invariant amplitude. Phys. Lett. B 1989, 224, 313-318. [CrossRef]

27. Rund, H. The Hamilton-Jacobi Theory in the Calculus of Variations: Its Role in Mathematics and Physics; New University Mathematics Series; Van Nostrand: Huntington, NY, USA, 1966.

28. Lanczos, C. The Variational Principles of Mechanics; Dover Books On Physics; Dover Publications: New York, NY, USA, 1970.

29. Licata, I. (Ed.) Beyond Peaceful Coexistence: The Emergence of Space, Time and Quantum; IMPERIAL COLLEGE PRESS, World Scientific Publishing Co. Pte. Ltd.: Singapore, 2016.

30. Green, M.B.; Schwarz, J.H.; Witten, E. Superstring Theory. Volume 1-Introduction; Cambridge University Press: Cambridge, UK, 1987.

31. Rizzuti, B.F.; Vasconcelos Júnior, G.F.; Resende, M.A. To square root the Lagrangian or not: An underlying geometrical analysis on classical and relativistic mechanical models. arXiv 2019, arXiv:1905.01177.

32. Hojman, S.; Harleston, H. Equivalent Lagrangians: Multidimensional case. J. Math. Phys. 1981, 22, 1414-1419. [CrossRef]

33. Baker, L.M.; Fairlie, D.B. Hamilton-Jacobi equations and brane associated Lagrangians. Nucl. Phys. B 2001, 596, $348-364$. [CrossRef]

34. Bouvier, P.; Maeder, A. Consistency of Weyl's Geometry as a Framework for Gravitation. ApESS 1978, 54, 497-508.

35. Maeder, A.; Bouvier, P. Scale invariance, metrical connection and the motions of astronomical bodies. AEA 1979, $73,82-89$.

36. Maeder, A.; Gueorguiev, V.G. The growth of the density fluctuations in the scale-invariant theory: One more challenge for dark matter. arXiv 2018, arXiv:1811.03495.

37. Landau, L.D.; Lifshitz, E.M. The Classical Theory of Fields, 4th ed.; Elsevier Science: Oxford, UK, 1975.

38. Rucker, R. Geometry, Relativity and the Fourth Dimension; Dover Books on Mathematics; Dover Publications: New York, NY, USA, 2012; pp. 1-160.

39. Magueijo, J.; Smolin, L. Lorentz Invariance with an Invariant Energy Scale. Phys. Rev. Lett. 2002, 88, 190403. [CrossRef] [PubMed]

40. Ragazzoni, R.; Turatto, M.; Gaessler, W. The Lack of Observational Evidence for the Quantum Structure of Spacetime at Planck Scales. ApJ 2003, 587, L1-L4. [CrossRef]

41. Fradkin, E.S.; Gitman, D.M. Path-integral representation for the relativistic particle propagators and BFV quantization. Phys. Rev. D 1991, 44, 3230-3236. [CrossRef] [PubMed]

42. Dirac, P.A.M. Generalized Hamiltonian Dynamics. Proc. R. Soc. Lond. Ser. A 1958, 246, 326-332. [CrossRef]

43. Teitelboim, C. Quantum mechanics on the gravitational field. Phys. Rev. D 1982, 25, 3159-3179. [CrossRef] 
44. Henneaux, M.; Teitelboim, C. Quantization of Gauge Systems; Princeton Paperbacks; Princeton University Press: Princeton, NJ, USA, 1994; pp. 1-520.

45. Sundermeyer, K. (Ed.) Constrained Dynamics; Lecture Notes in Physics; Springer: Berlin, Germany, 1982; Volume 169. [CrossRef]

46. de León, M.; Marrero, J.C.; de Diego, D.M.; Vaquero, M. On the Hamilton-Jacobi theory for singular lagrangian systems. J. Math. Phys. 2013, 54, 032902. [CrossRef]

47. Deriglazov, A.A. On singular Lagrangian underlying the Schrödinger equation. Phys. Lett. A 2009, 373, 3920-3923. [CrossRef]

48. Page, D.N.; Wootters, W.K. Evolution without evolution: Dynamics described by stationary observables. Phys. Rev. D 1983, 27, 2885-2892. [CrossRef]

49. Géhéniau, J.; Prigogine, I. The birth of time. Found. Phys. 1986, 16, 437-443. [CrossRef]

50. Gaioli, F.H.; Garcia-Alvarez, E.T. The problem of time in parametrized theories. Gen. Relativ. Gravit. 1994, 26, 1267-1275. [CrossRef]

51. Elze, H.T.; Schipper, O. Time without time: A stochastic clock model. Phys. Rev. D 2002, 66, 044020. [CrossRef]

52. Elze, H.T. Quantum Mechanics Emerging from “Timeless" Classical Dynamics. In Trends in General Relativity and Quantum Cosmology; Benton, C.V., Ed.; Nova Science Publ.: Hauppauge, NY, USA, 2006; pp. 79-101.

53. Albrecht, A.; Iglesias, A. Clock ambiguity and the emergence of physical laws. Phys. Rev. D 2008, 77, 063506. [CrossRef]

54. Bicego, A. On a probabilistic definition of time. arXiv 2010, arXiv:1010.2968.

55. Viznyuk, S. Time as a parameter of statistical ensemble. arXiv 2011, arXiv:1110.3296.

56. Bojowald, M.; Höhn, P.A.; Tsobanjan, A. An effective approach to the problem of time. Class. Quantum Gravity 2011, 28, 035006. [CrossRef]

57. Prati, E. Generalized clocks in timeless canonical formalism. J. Phys. Conf. Ser. 2011, 306, 012013. [CrossRef]

58. Wetterich, C. Probabilistic Time. Found. Phys. 2012, 42, 1384-1443. [CrossRef]

59. Barbour, J.; Lostaglio, M.; Mercati, F. Scale anomaly as the origin of time. Gen. Relativ. Gravit. 2013, 45, 911-938. [CrossRef]

60. Vaccaro, J.A. T Violation and the Unidirectionality of Time: Further Details of the Interference. Found. Phys. 2015, 45, 691-706. [CrossRef]

61. Marletto, C.; Vedral, V. Evolution without evolution and without ambiguities. Phys. Rev. D 2017, 95, 043510. [CrossRef]

62. Elçi, A. Time in classical and in quantum mechanics. J. Phys. Math. Gen. 2010, 43, 285302. [CrossRef]

63. Renner, R.; Stupar, S. Time in Physics; Tutorials, Schools, and Workshops in the Mathematical Sciences; Springer International Publishing AG: Cham, Switzerland, 2017.

64. Velev, M.V. Relativistic mechanics in multiple time dimensions. Phys. Essays 2012, 25, 403-438. [CrossRef]

65. Craig, W.; Weinstein, S. On determinism and well-posedness in multiple time dimensions. Proc. R. Soc. Lond. Ser. A 2009, 465, 3023-3046. [CrossRef]

66. Carroll, S.M.; Chen, J. Spontaneous Inflation and the Origin of the Arrow of Time. arXiv 2004, arXiv:hep-th/0410270.

67. Carroll, S.M.; Chen, J. Does inflation provide natural initial conditions for the universe. Gen. Relativ. Gravit. 2005, 37, 1671-1674. [CrossRef]

68. Gurzadyan, V.G.; Sargsyan, S.; Yegorian, G. On the time arrows, and randomness in cosmological signals. Eur. Phys. J. Web Conf. 2013, 58, 02005. [CrossRef]

69. Page, D.N. Inflation does not explain time asymmetry. Nature 1983, 304, 39-41. [CrossRef]

70. Davies, P.C.W. Inflation in the universe and time asymmetry. Nature 1984, 312, 524-527. [CrossRef]

71. Lawrie, I.D. Time evolution in quantum cosmology. Phys. Rev. D 2011, 83, 043503. [CrossRef]

72. Gonzalez-Ayala, J.; Cordero, R.; Angulo-Brown, F. Is the $(3+1)$-d nature of the universe a thermodynamic necessity? EPL (Europhys. Lett.) 2016, 113, 40006. [CrossRef]

73. Mukohyama, S.; Uzan, J.P. From configuration to dynamics: Emergence of Lorentz signature in classical field theory. Phys. Rev. D 2013, 87, 065020. [CrossRef]

74. Pradhan, R.K. Observed Spacetime Dimensionality from Fundamental Principles. arXiv 2013, arXiv:1303.5634.

75. Arkani-Hamed, N.; Cohen, A.G.; Georgi, H. (De)Constructing Dimensions. Phys. Rev. Lett. 2001, 86, 4757-4761. [CrossRef]

76. Tegmark, M. LETTER TO THE EDITOR: On the dimensionality of spacetime. Class. Quantum Gravity 1997, 14, L69-L75. [CrossRef]

77. Callender, C. Answers in search of a question: 'proofs' of the tri-dimensionality of space. Stud. Hist. Philos. Mod. Phys. 2005, 36, 113-136. [CrossRef]

78. Bekenstein, J.D. Relation between physical and gravitational geometry. Phys. Rev. D 1993, 48, 3641-3647. [CrossRef] [PubMed]

79. Stückelberg de Breidenbach, E.C.G.; Scheurer, P.B. Thermocinétique Phénoménologique Galiléenne; Lehrbücher und Monographien aus dem Gebiete der exakten Wissenschaften; Mathematische Reihe, Birkhäuser: Basel, Switzerland; Stuttgart, Germany, 1974; p. 253.

80. Pavšič, M. Klein-Gordon-Wheeler-DeWitt-Schrödinger equation. Phys. Lett. B 2011, 703, 614-619. [CrossRef]

81. Bojowald, M.; Höhn, P.A.; Tsobanjan, A. Effective approach to the problem of time: General features and examples. Phys. Rev. D 2011, 83, 125023. [CrossRef]

82. Gryb, S.; Thébault, K. The Role of Time in Relational Quantum Theories. Found. Phys. 2012, 42, 1210-1238. [CrossRef]

83. Schwinger, J. Quantized Gravitational Field. Phys. Rev. 1963, 130, 1253-1258. [CrossRef] 
84. Pavsic, M. The Landscape of Theoretical Physics: A Global View: From Point Particles to the Brane World and Beyond. In Search of a Unifying Principle; Kluwer Academic: Dordrecht, The Netherlands; Boston, MA, USA, 2001.

85. Kuwabara, R. Time-dependent mechanical symmetries and extended Hamiltonian systems. Rep. Math. Phys. 1984, 19, 27-38. [CrossRef]

86. Landau, L.; Lifshitz, E. Quantum Mechanics: Non-Relativistic Theory; Course of Theoretical Physics; Elsevier Science: Amsterdam, The Netherlands, 1981.

87. Antoine, J.P. Beyond Hilbert space: RHS, PIP and all that. J. Phys. Conf. Ser. 2019, 1194, 012007. [CrossRef]

88. Deriglazov, A.A.; Pupasov-Maksimov, A.M. Frenkel electron on an arbitrary electromagnetic background and magnetic Zitterbewegung. Nucl. Phys. B 2014, 885, 1-24. [CrossRef]

89. Deriglazov, A.A.; Pupasov-Maksimov, A.M. Geometric Constructions Underlying Relativistic Description of Spin on the Base of Non-Grassmann Vector-Like Variable. SIGMA 2014, 10, 012. [CrossRef]

90. Deriglazov, A.A.; Pupasov-Maksimov, A.M. Lagrangian for Frenkel electron and position's non-commutativity due to spin. arXiv 2013, arXiv:1312.6247.

91. Deriglazov, A.A.; Gitman, D.M. Classical Description of Spinning Degrees of Freedom of Relativistic Particles by Means of Commuting Spinors. Mod. Phys. Lett. A 1999, 14, 709-720. [CrossRef] 